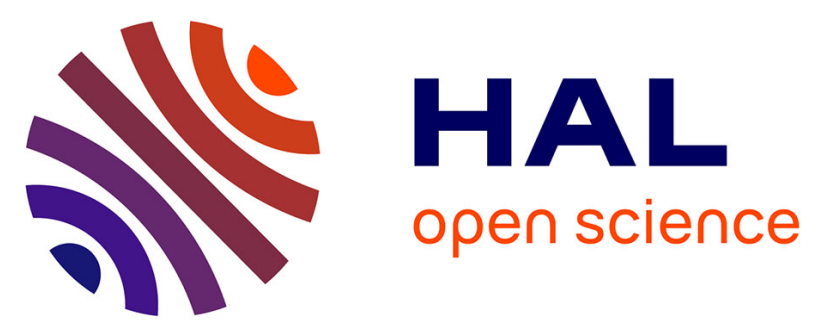

\title{
Development and Application of Bivariate 2D-EMD for the Analysis of Instantaneous Flow Structures and Cycle-to-Cycle Variations of In-cylinder Flow
}

Mehdi Sadeghi, Karine Truffin, Brian Peterson, Benjamin Böhm, Stéphane Jay

\section{To cite this version:}

Mehdi Sadeghi, Karine Truffin, Brian Peterson, Benjamin Böhm, Stéphane Jay. Development and Application of Bivariate 2D-EMD for the Analysis of Instantaneous Flow Structures and Cycle-toCycle Variations of In-cylinder Flow. Flow, Turbulence and Combustion, 2021, 106 (1), pp.231-259. 10.1007/s10494-020-00197-z . hal-03136997

\section{HAL Id: hal-03136997 \\ https://hal-ifp.archives-ouvertes.fr/hal-03136997}

Submitted on 10 Feb 2021

HAL is a multi-disciplinary open access archive for the deposit and dissemination of scientific research documents, whether they are published or not. The documents may come from teaching and research institutions in France or abroad, or from public or private research centers.
L'archive ouverte pluridisciplinaire HAL, est destinée au dépôt et à la diffusion de documents scientifiques de niveau recherche, publiés ou non, émanant des établissements d'enseignement et de recherche français ou étrangers, des laboratoires publics ou privés. 
Noname manuscript No.

(will be inserted by the editor)

\title{
Development and application of Bivariate 2D-EMD for the analysis of instantaneous flow structures and cycle-to-cycle variations of in-cylinder flow
}

\author{
Mehdi Sadeghi · Karine Truffin \\ (corresponding author) • Brian \\ Peterson - Benjamin Böhm · Stephane \\ Jay
}

Received: date / Accepted: date

\begin{abstract}
The bivariate two dimensional empirical mode decomposition (Bivariate 2D-EMD) is extended to estimate the turbulent fluctuations and to identify cycle-to-cycle variations (CCV) of in-cylinder flow. The Bivariate 2DEMD is an adaptive approach that is not restricted by statistical convergence criterion, hence it can be used for analyzing the nonlinear and non-stationary phenomena. The methodology is applied to a high-speed PIV dataset that measures the velocity field within the tumble symmetry plane of an optically accessible engine. The instantaneous velocity field is decomposed into a finite number of $2 \mathrm{D}$ spatial modes. Based on energy considerations, the in-cylinder flow large-scale organized motion is separated from turbulent fluctuations. This study is focused on the second half of the compression stroke. For most of the cycles, the maximum of turbulent fluctuations is located between 50 and 30 crank angle degrees (CAD) before top dead center (TDC). In regards to the phase-averaged velocity field, the contribution of CCV to the fluctuating kinetic energy is approximately $55 \%$ near TDC.
\end{abstract}

Keywords In-cylinder flow · Bivariate 2D-EMD · Turbulence · Large-scale organized structure

\footnotetext{
Mehdi Sadeghi · Karine Truffin · Stephane Jay

IFP Energies nouvelles, 1 et 4 avenue du Bois-Préau, 92852 Rueil-Malmaison, France

E-mail: mehdi.sadeghi-ghahi@ifpen.fr

E-mail: karine.truffin@ifpen.fr

Brian Peterson

School of Engineering, Institute of Multiscale Thermofluids, University of Edinburgh,

The King's Buildings, Mayfield Road, Edinburgh, EH93FD, UK

Benjamin Böhm

Reaktive Strömungen und Messtechnik, Technische Universität Darmstadt, Otto-Berndt-

Straße 3, 64287 Darmstadt, Germany
} 


\section{Introduction}

The in-cylinder flow plays a major role in the performance of internal combustion engines. Air-fuel mixing process, combustion development, auto-ignition, filling-empty efficiency and pollutant emissions are all affected by air motion $[17,30,39,51]$. The in-cylinder turbulent flow is inherently unsteady since the flow pattern changes during individual cycles and the mean flow varies from one cycle to another. Such large-scale cycle-to-cycle variation (CCV) of the flow has a negative effect on combustion process and can cause engine knock, missfire, excessive noise and emissions [33].

The turbulent fluctuations are considered to be different from the large-scale variations of the flow. The turbulent eddies cause small-scale mixing, moreover the high level of turbulent kinetic energy enhances flame propagation and reduces the duration of combustion process. Hence the separation of random turbulent fluctuations from the larger-scale coherent structures, that are associated with CCV, is of crucial importance in engine development. Different decomposition methods exist in the literature. A non exhaustive list is presented in the diagram of Fig. 1. These approaches can be generalized into two main categories: statistical and non-statistical. The statistical approaches require several instantaneous velocity fields for every crank angle degree (CAD) such as cyclic or moving averaging techniques [31,44]. The wellknown Proper Orthogonal Decomposition (POD) is another statistical method that was proposed by Lumley for turbulent flows [28]. It is a linear procedure that decomposes the velocity field into a set of orthogonal basis functions (modes) in which the flow structures containing most of the kinetic energy are reconstructed by the first few modes. It is an adaptive method in which the basis functions are computed from the data itself. POD has been commonly applied to in-cylinder flow in order to extract the dominant structures and study their CCV $[5,9,13,27]$. However in POD filtering, the separation between mean flow and turbulent fluctuations is performed by truncating the series of the modes and this requires an ad hoc criterion [7,40]. Furthermore, Independent Component Analysis (ICA) can be coupled to POD in order to analyze in-cylinder flow structures and to provide information about CCV [3]. Recently, Cluster-based analysis was proposed for identification of CCV [6]. Although this approach extends the POD analysis, it can be applied on any periodical driven flow at large Reynolds number. Dynamical Mode Decomposition (DMD) is an alternative statistical method for analyzing nonlinear turbulent flows [43]. It was applied to in-cylinder velocity fields resolved by large eddy simulation (LES) in order to extract the spatial flow structures at certain frequencies [42]. All aforementioned methods provide a statistical description of the flow structures, hence they are not appropriate for analyzing non-stationary dynamics.

Non-statistical methods can include approaches such as Wavelet Analysis [48]. It is suitable in order to study unsteady flows (as in-cylinder flow), however the results depend on the prior defined basis function (mother wavelet) in the decomposition process. Fourier transform can also be used to separate in- 
cylinder mean flow from fluctuating component. However the decomposition is based on the harmonic functions, in addition like most of turbulent flows, the choice of a cut-off frequency/length remains a complex subject $[8,26]$.

In addition, various spatial filter techniques such as Gaussian kernel exist, which distinguish CCV from turbulent fluctuations [19,38]. However, the results depend again on the filter size. Lagrangian finite time techniques can also be used to detect precisely the evolution of spatial coherent structures and to investigate the unsteady flow separation. This has been applied to in-cylinder velocity fields to extract turbulent fluctuations at the end of compression stroke [46]. However, the realization of this technique is quite a complicated task.

Variational Mode Decomposition (VMD) can be an alternate candidate for analyzing non-stationary phenomena [10]. It is a non-recursive method where the modes are extracted concurrently. This method requires a prior information about mode numbers, as many segmentation algorithms, in order to avoid over/under decomposition problem.

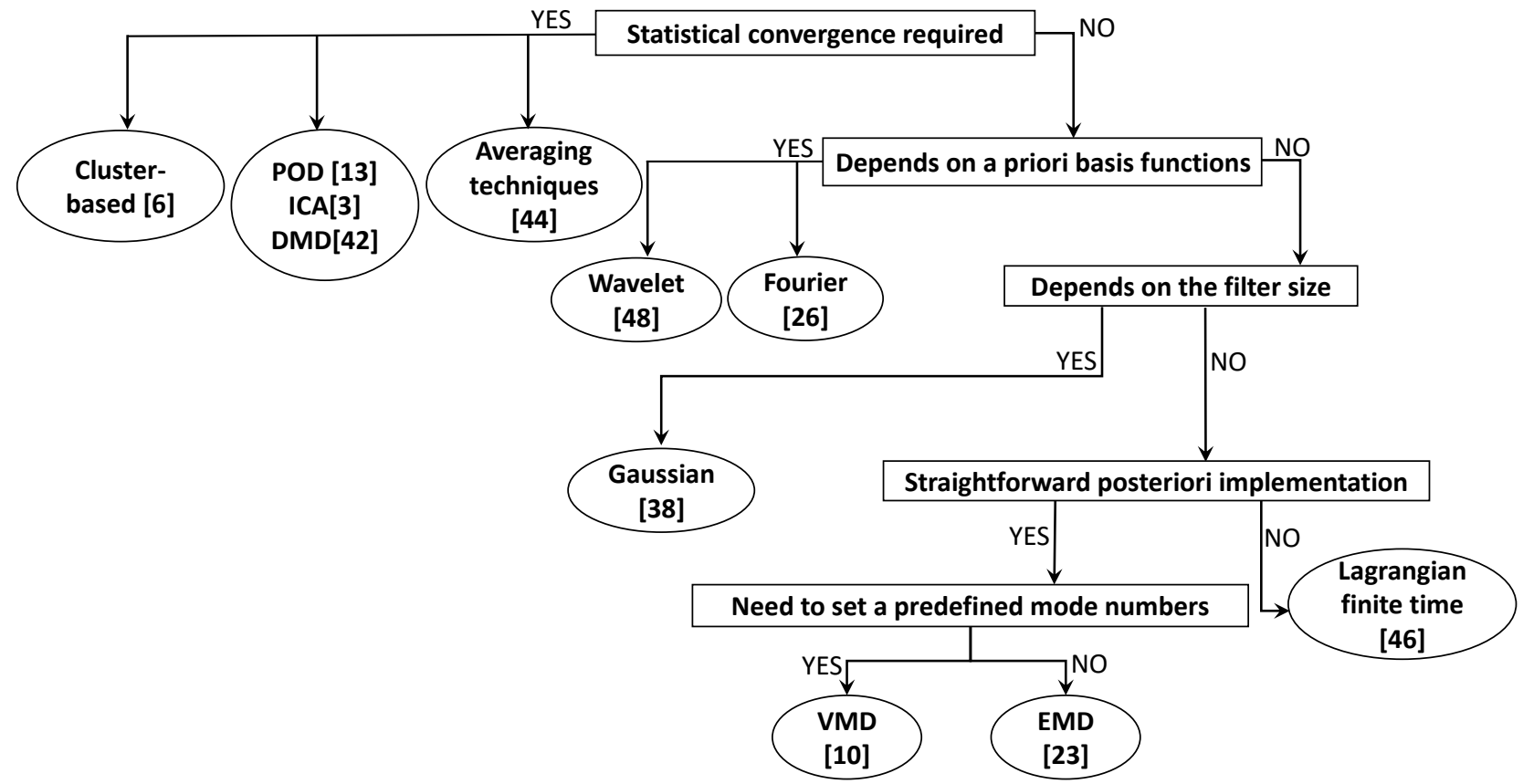

Fig. 1: Diagram presenting decomposition methods (non exhaustive) for analyzing in-cylinder velocity fields sorted following several criteria 
Empirical Mode Decomposition (EMD) is a fully data-driven method for analyzing the nonlinear and non-stationary phenomena that was introduced by Huang et al. [23]. It decomposes any complicated dataset into a finite number of oscillatory modes, called Intrinsic Mode Functions (IMFs), with significant different bandwidth frequency. The IMF has two properties: in the whole dataset, the number of extrema and zero-crossing are equal or differs at most by one. In addition, it has a zero mean envelope defined by the local maxima and minima. It should be emphasized that unlike any simple harmonic function, Fourier transform, the amplitude and frequency of the IMF varies as a function of time.

EMD has encountered considerable success in atmospheric physics, geophysics and brain-computer interfaces $[12,36,49]$. In fluid mechanics, Huang et al. [22] used EMD and a Hilbert transform for identification of different events in Stokes and nonlinear water waves. Huang et al. [24] applied EMD on homogeneous turbulence time series to investigate turbulent scaling intermittency, however such applications are restricted to the temporal analysis of the velocity fields at one point. Recently, Sadeghi et al. [41] proposed Bivariate Two dimensional EMD (Bivariate 2D-EMD) in order to separate spatial large-scale organized motion from homogeneous and isotropic turbulent flow. Although EMD analysis has never been applied to confined, unsteady turbulent flows, as those encountered in piston engines, it appears to be promising for engine flow analysis. One of its obvious advantages is the ability to decompose the flow structure on a single instantaneous velocity field. Therefore such an approach appears appropriate for analyzing in-cylinder flows within individual cycles under transient and steady engine operations.

This work presents the extension and application of Bivariate 2D-EMD for the analysis of in-cylinder flow velocity fields obtained from planar high-speed PIV. Bivariate 2D-EMD is used to separate large-scale organized motions from small-scale turbulent fluctuations. The former is classified as the low spatial frequency part of the flow, while the latter is the high spatial frequency part. This analysis allows one to estimate in-cycle turbulent fluctuations based on the velocity field of an individual cycle. In addition, the variability of lowspatial frequency flows from the phase-averaged flows, provides the ability to study the cycle-to-cycle variations of the organized flow motion.

Moreover, the use of this method that can improve the center identification of the tumble motion, is investigated. The tumble is the in-cylinder large-scale rotational motion in spark-ignition engine, the generating and control of this flow have a considerable importance for engine designer.

\section{Bivariate 2D-EMD}

Given an arbitrary one dimensional and mono-component signal $x(t)$, the algorithm of standard EMD can be summarized as follows:

1. Identify all local extrema of the variable $x(t)$. 
2. Interpolate (using local spline interpolation) between all the minima (resp. maxima) to obtain the signal envelope passing through the minima, $e_{\min }(t)$ (resp. $\left.e_{\max }(t)\right)$.

3. Compute the local mean $m(t)=\left(e_{\min }(t)+e_{\max }(t)\right) / 2$.

4. Subtract the local mean from the signal to obtain the modulated oscillation, local detail $h(t)=x(t)-m(t)$.

5. If $h(t)$ satisfies the stopping criterion [47], then it is an IMF: $I M F(t)=$ $h(t)$, otherwise set $x(t)=h(t)$ and repeat the process from step 1 .

The above procedure is called Sifting process and once the first IMF is obtained, it is applied in the iterative way to the residual $r(t)=x(t)-I M F(t)$ to extract remaining modes, called IMFs. The decomposition progresses until the residual (last mode) becomes a monotonic function, such that the original signal is written as: $x(t)=\sum_{i=1}^{N} I M F_{i}(t)$. In this way EMD gradually decomposes any given signal, and the first IMF contains the highest frequency (finest scale) and the last IMF represents the signal tendency. For instance, the decomposition of an experimental temporal turbulent velocity signal by EMD is presented in Fig. 2.



Fig. 2: Example of EMD analysis of a turbulent velocity signal (First row) and the corresponding IMFs, the unit is $\mathrm{ms}^{-1}$

The Bivariate 2D-EMD approach decomposes a two-components (bivariate) signal in 2D spatial field [41]. This method is based on Adaptive-Projection Intrinsically Transformed Multivariate EMD (APIT-MEMD) that processes temporal multi-components signals [20]. Also in order to suppress the modemixing problem, the noise-assisted modes are considered in the decomposition 
process $[37,45]$. The mode-mixing refers to the presence of single scale in different modes or a single mode containing several various scales. The added White Gaussian Noise (WGN) provides the uniform reference frame in the time-frequency space. When the signal is added to WGN, the intrinsic signal oscillations at different scales are automatically associated with the similar scales of reference background established by white noise. In Noise assisted APIT-MEMD (NA-APIT-MEMD) [20], the WGN is considered as an adjacent subspace and then the composite signal is decomposed by APIT-MEMD. Finally the IMF components corresponding to WGN are discarded thus leading to a set of IMFs associated with only original input signal. The WGN amplitude is set close to those of the input signal components in order to avoid power imbalance. In addition, a single realization of $l$-channel WGN in the decomposition leads to rather different outputs for different trials. Therefore, an ensemble approach should be considered in which the input multivariate signal is decomposed several times (NE). It means every time a different set of $l$-channel noise is used. Then the set of multivariate IMFs obtained from the decompositions are ensemble averaged to obtain the final IMFs. This method is called Ensemble NA-APIT-MEMD.

In order to decompose the two-components $2 \mathrm{D}$ signal as an instantaneous 2D velocity field with horizontal and vertical components using Bivariate 2D-EMD analysis, the signal is considered as $f(m, n)$ gridded data. At first step, the ensemble NA-APIT-MEMD that simultaneously decomposes the two components of the velocity field, is applied to each row of the velocity field that leads to $K$ horizontal components. It should be noted that the $U$ and $V$ components of the velocity field are processed in a "coupled way". Afterwards, it is applied in the vertical direction on the results of decomposition at the first step (i.e., on each column of the horizontal components), generating $J$ vertical components, leading to a total number of $J \times K$ two-dimensional components (pseudo IMFs). To illustrate, an instantaneous 2D in-cylinder velocity field at 100 crank angle degrees before compression stroke top dead center (in this study noted $100 \mathrm{CAD}$ ) is shown in Fig. 3a. The horizontal component of this velocity field, $U$ and the corresponding two-dimensional components i.e., the horizontal components of the pseudo IMFs are presented in Fig. 3b and Fig.4, respectively. By careful consideration some oriented scales and similar characteristics in different pseudo IMFs can be observed, for instant the horizontal (vertical) scales of each row (column) increase as $k(j)$ increases. 


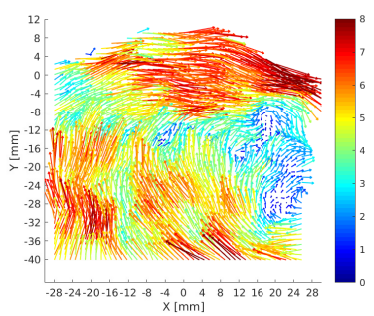

(a)



(b)

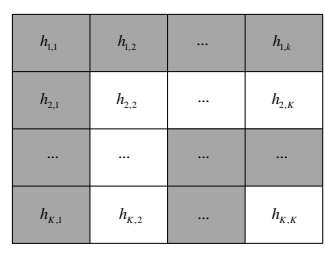

(c)

Fig. 3: (a) In-cylinder instantaneous velocity field at $100 \mathrm{CAD}$, the unit is $\mathrm{ms}^{-1}$, (b) $U$, horizontal component of the velocity field, (c) The scheme of combination strategy for different components resulting from applying Ensemble NA-APIT-MEMD in two orthogonal directions

The final $i$-th bivariate and bidimensional IMF $\left(\mathrm{BIMF}_{i}\right)$ is obtained by using a combination strategy [50]; $\mathrm{BIMF}_{i}=\sum_{k=i}^{K} h_{i, k}+\sum_{j=i+1}^{J} h_{j, i}$, where $h_{j, k}$ of size $(m, n)$ denotes the pseudo IMF that is located in the $j$-th row and $k$-th column. Figure 3c depicts this strategy in which the neighbor pseudo IMF with the same shade are summed in order to obtain the final modes. Figure 5 presents only the horizontal components of the final BIMFs that correspond to the modes of $U$. It is observed that the range of spatial frequencies of the fluctuations decreases from the first to the last mode and the latter represents the trend of the velocity field in the horizontal direction. The modes (BIMFs) that contain the two components of the velocity field $(U, V)$ are presented in Fig. 6. For Bivariate 2D-EMD analysis, the noise assisted process was performed by one WGN for which the variance was set to the mean variance of the input signal components. In this manner, the noise itself does not generate power imbalance between input signal components and the number of ensemble realizations, NE is 100 . 




Fig. 4: The horizontal components of the pseudo IMFs corresponding to $U$ velocity field, that are obtained by the application of Ensemble NA-APITMEMD in the horizontal and vertical directions of the velocity field in Fig. 3a, the scale in each panel is different

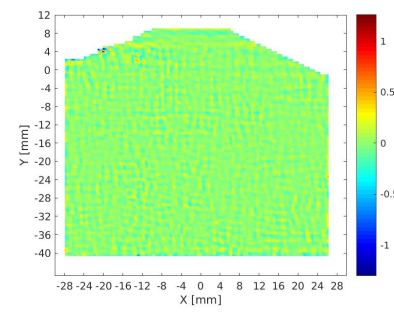

(a)

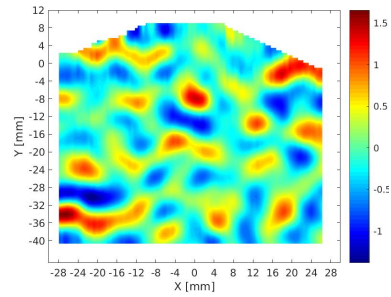

(d)

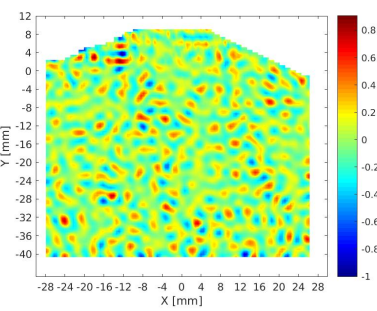

(b)

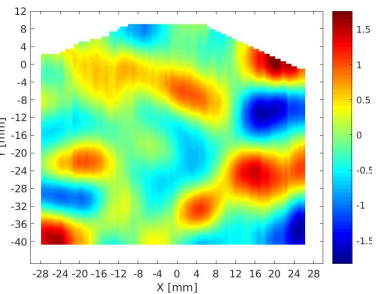

(e)

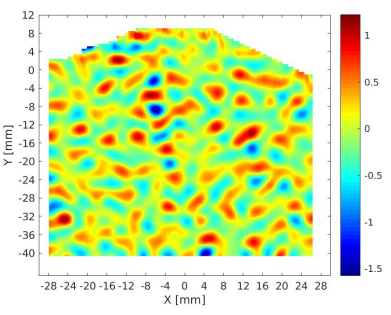

(c)

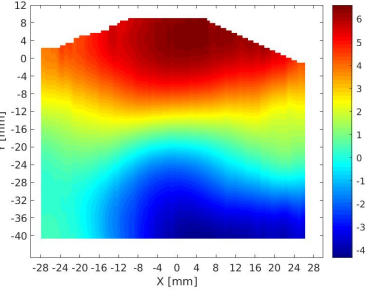

(f)

Fig. 5: The horizontal components of the final BIMFs of the velocity field, (a-f) from the first to the last mode, respectively, the unit is $\mathrm{ms}^{-1}$ 


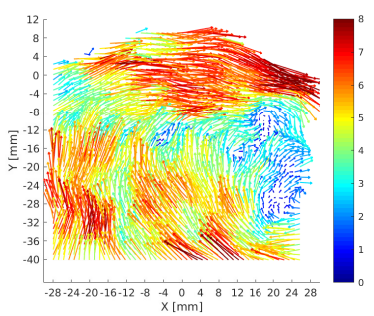

(a)

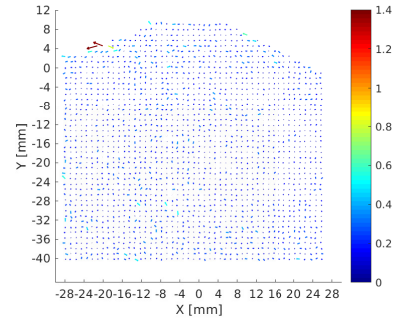

(b)

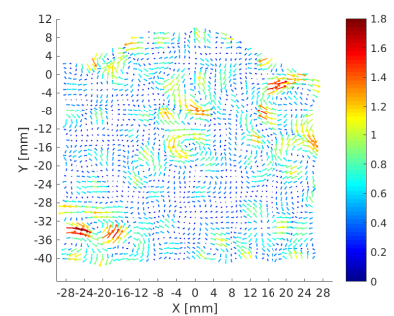

(e)

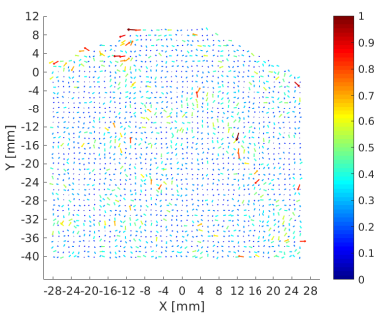

(c)

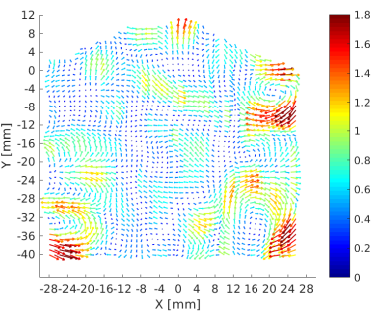

(f)



(d)



(g)

Fig. 6: (a) In-cylinder instantaneous velocity field at $100 \mathrm{CAD}$ (presented in Fig. 3a) and (b-g) Its BIMFs from the first to last mode, respectively. The unit is $\mathrm{ms}^{-1}$ and every two vectors are depicted

In the present study the Bivariate 2D-EMD is extended in order to be applied to complex and time-dependent geometries as encountered in the engine configurations in which the size of velocity field changes due to the piston motion. Two main difficulties are identified: determination of the global number of "pseudo IMFs" in each direction and also the total number of the "final modes (BIMFs)" for each velocity field. These issues and proposed solutions are detailed in the following.

Firstly, the total number of the modes obtained by EMD analysis for 1D signal is typically at most $O\left(\log _{2} N\right)$ for $N$ data points. So when the velocity field has a polygon shape (similar to engine flows with a pentroof cylinder head), the number of the modes can vary from one row (resp. column) of data to the others. Moreover, such a situation can be encountered even for a square velocity field due to the presence of different scales of fluctuations in the local 
velocity i.e., signal complexity. In these cases, the total number of the pseudo IMFs i.e., $J \times K$ is given by the number of modes for the row (resp. column) of data that contains most of the modes. The last mode of the row (resp. column) of data that has a fewer modes should be considered as the $K$-th (resp. $J$-th) mode in order to have the meaningful trend of the analyzed signal in the final BIMF. This trend contains the lowest spatial frequency of the signal for which the faster fluctuations are superimposed on. The other modes of this row (resp. column) data are also organized in the descending order for construction of the $h_{j, k}$ components.

Secondly, considering the aforementioned combination strategy for 2D signal, the total number of the final modes (BIMFs) is driven by the direction that has the smallest length. This fact can lead to less physical significant modes when the signal feature (i.e., flow structures) are more complex in the other direction. Such a situation can be encountered for flows dealing with time-varying geometries as in the case of in-cylinder flows as the piston moves. Therefore, in order to equally weight the decomposition in both directions and not to impose a prior preferential direction, the signal in the smaller direction is interpolated on the grid with the same number points as in the larger direction. In this manner, the dimension of the original $2 \mathrm{D}$ signal is not changed contrary to the phase-invariant POD method [13]. In this latter approach, the velocity fields are stretched in the cylinder axis direction to obtain data on a fixed grid that makes it possible to study the velocity field evolution in terms of the POD basis functions. In the present grid interpolation approach, the verification of the signal's energy (the mean kinetic energy of the velocity field) for several instantaneous in-cylinder flow (100, 50, $25 \mathrm{CAD})$, before and after different interpolation methods has been performed. The results shows an error of $0.7 \%$ in the case of linear interpolation scheme which is less than ones obtained by natural or cubic scheme. The procedure of Bivariate 2D-EMD analysis to separate the large-scale organized flow motion from turbulence is summarized as follows:

1. Interpolate the input $2 \mathrm{D}$ signal in the smaller direction on the grid with the same number of the points as in the larger direction.

2. Apply the Ensemble NA-APIT-MEMD to each row (or column) of the bivariate 2D signal, i.e., decomposition along one dimension.

3. Apply the Ensemble NA-APIT-MEMD to each column (or row) of the results of the decomposition from step 2, i.e., decomposition along the second dimension.

4. Use the combination strategy to obtain final bivariate 2D modes.

5. Calculate the kinetic energy of each mode following Eq. (3).

6. Separate the flow large-scale organized motion (i.e., the last IMF) from turbulent fluctuations based on energy considerations. Indeed the last IMF provides the evolution of the large-scale motion.

The efficiency of this approach was also assessed through the decomposition of different velocity fields. For instance a known synthetic Lamb-Oseen vortex, 
$\mathrm{U}_{i}(x, y)$ that mimics coherent structures is perturbed by a section of an experimental homogeneous isotropic turbulent (HIT) velocity field, $\mathrm{P}(x, y)$, by spatial resolution of $0.16 \mathrm{~mm}[16]$. After the perturbed velocity field, $\mathrm{U}_{p}(x, y)$, is decomposed by Bivariate 2D-EMD and based on energy consideration, presented in Sec. 3, the synthetic vortex is recovered. The resulting flow field, $\mathrm{U}_{r}(x, y)$, from this analysis is presented in Fig.7. The relative mean square error between the recovered vortex and the initial one is 6.74 and $6.3 \%$ without and with interpolation respectively. More explications about the influence of the velocity field interpolation on the final modes and the separation of different parts of the flow are presented in appendix.

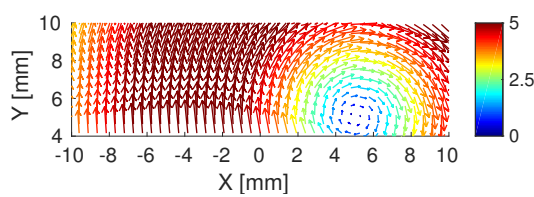

(a) $\mathrm{U}_{i}(x, y)$, Large-scale synthetic flow

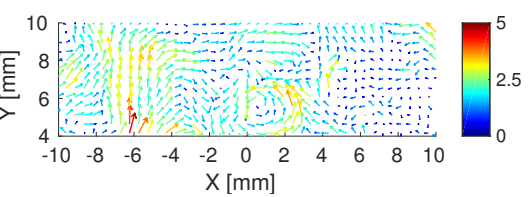

(b) $\mathrm{P}(x, y)$, Small-scale structures

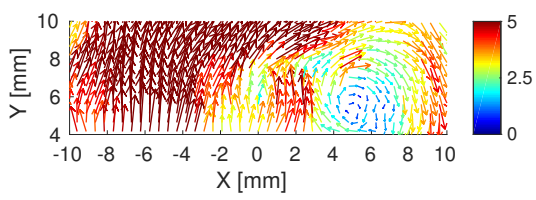

(c) $\mathrm{U}_{p}(x, y)=\mathrm{U}_{i}(x, y)+\mathrm{P}(x, y)$

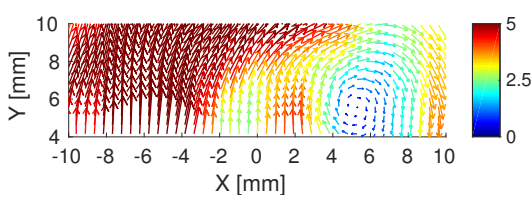

(d) $\mathrm{U}_{r}(x, y)$



(e) $\mathrm{U}_{r}(x, y)$

Fig. 7: (a) A synthetic Lamb-Oseen vortex, (b) A section of an experimental HIT velocity field as a perturbation, (c) Perturbed velocity field, (d-e) Recovered velocity fields (Large-scale structure) without and with interpolation, respectively. The unit is $\mathrm{ms}^{-1}$ and every two vectors are displayed

\section{Application of Bivariate 2D-EMD on in-cylinder flow dataset}

3.1 High speed planer PIV and optical engine

The PIV measurements evaluated in this study were performed in an optically accessible single-cylinder spark-ignition wall-guided engine. The optical engine and PIV measurements have been previously described elsewhere $[2,15,52]$ and a brief description is provided as follows. The optical engine features a twin-cam, overhead valve pentroof cylinder head, a $55 \mathrm{~mm}$ height quartz-glass 
cylinder liner with $8 \mathrm{~mm}$ window extension into the pentroof, and a Bowditch piston arrangement with a flat quartz-glass piston crown window. The cylinder head is equipped with a side-mounted injector, which remained inactive for the experiments, and a centrally mounted spark plug, which was replaced with a threaded plug whose surface remained flush with the cylinder head surface. For the measurements used in this study, the engine operated at $800 \mathrm{rpm}$ under motored conditions, Table 1 provides relevant engine details and operating parameters.

Table 1: Engine operating parameters

\begin{tabular}{ll}
\hline Engine speed & $800 \mathrm{rpm}$ \\
Bore/Stroke & $86 / 86 \mathrm{~mm}$ \\
Clearance height & $2.6 \mathrm{~mm}$ \\
Compression ratio & $8.5: 1$ \\
Displacement volume & $499 \mathrm{~cm}^{3}$ \\
Average intake pressure & $0.95 \mathrm{bar}$ \\
Average exhaust pressure & $1 \mathrm{bar}$ \\
Intake air temperature & $\sim 23^{\circ} \mathrm{C}$ \\
Cylinder head coolant temperature & $\sim 60^{\circ} \mathrm{C}$ \\
Intake valve open & $325^{\circ}$ aTDC $a$ \\
Intake valve close & $125^{\circ} \mathrm{bTDC}{ }^{a}$ \\
Exhaust valve open & $105^{\circ}$ aTDC \\
Exhaust valve close & $345^{\circ} \mathrm{bTDC}$ \\
\hline$a$ TDC denotes top dead center of compression stroke, \\
a and b stands for after and before
\end{tabular}

High-speed PIV measurements were performed in the optical engine to capture the temporal evolution of the two-dimensional, two-component (2D2C) velocity field throughout the entire engine cycle (i.e., 719 consecutive CADs). A frequency-doubled $\mathrm{Nd}: \mathrm{YVO}_{4}$ dual-cavity laser and 12-bit CMOS camera were synchronized to the engine at $4.8 \mathrm{kHz}$ to provide 2D PIV images every crank-angle. A homemade programmable timing unit, including two signal generators (Quantum Composers), was used to specify independently the time separation between the double-pulsed laser pulses throughout the engine cycle. This provided an optimization of the timing between PIV frames on a crank-angle resolved basis, which effectively increases the dynamic range of the PIV measurements $[1,34]$. Silicone oil droplets $(\sim 1 \mu \mathrm{m}$ diameter $)$ were used as the PIV seeding medium, and were introduced into the intake air via a droplet seeder.

The high-speed PIV images resolved the velocity field within a $54 \times 54 \mathrm{~mm}^{2}$ region (1.0 mm light sheet thickness) centered within the central symmetry plane (i.e., $\mathrm{z}=0 \mathrm{~mm}$ ). PIV images were processes with a commercial software (DaVis, LaVision). A multi-pass cross-correlation algorithm with decreasing window size $(96 \times 96$ to $32 \times 32$ pixels $)$ and $75 \%$ window overlap was used for PIV processing. The PIV measurements have a spatial resolution of 2.25 $\mathrm{mm}$ based on the final interrogation window size $(32 \times 32$ pixels $)$ and a vector spacing of $0.56 \mathrm{~mm}$. Further details of the PIV measurements and their uncertainty can be found in $[2,52]$. 


\subsection{Bivariate 2D-EMD analysis}

We intend to provide an alternate way for triple decomposition of the velocity field in the case of in-cylinder flows [21], in which the local instantaneous velocity at crank angle $\theta$ during cycle $i$ is presented as

$$
\mathbf{U}(\theta, i)=\mathbf{U}_{\mathbf{E A}}(\theta)+\mathbf{u}^{\mathbf{c}}(\theta, i)+\mathbf{u}^{\mathbf{r}}(\theta, i),
$$

The three terms on the right hand side are respectively the ensemble (phase)averaged velocity; $\mathbf{U}_{\mathbf{E A}}(\theta)=\langle\mathbf{U}(\theta, i)\rangle$, the coherent fluctuations associated with cycle-to-cycle variation of the organized motion (CCV) and the turbulent fluctuations.

In this study, Bivariate 2D-EMD is applied to the instantaneous velocity fields in order to separate the large-scale organized motion i.e., the low spatial frequency part (Lf) from the turbulent fluctuations i.e., the high spatial frequency part (Hf). Hence, the instantaneous velocity can be represented as

$$
\mathbf{U}(\theta, i)=\mathbf{U}_{\mathbf{L f}}(\theta, i)+\mathbf{U}_{\mathbf{H f}}(\theta, i),
$$

By such a definition, one can have access to the particular cycle bulk flow and the velocity fluctuations that are considered as the turbulence. The difference between the flow low frequency part and the phase-averaged velocity field corresponds to the cycle-to-cycle variation of the large-scale motion, described as $\mathbf{U}_{\mathbf{c}}(\theta, i)=\mathbf{U}_{\mathbf{L f}}(\theta, i)-\mathbf{U}_{\mathbf{E A}}(\theta)$. As one can observe in Fig. 6, the instantaneous velocity field at $100 \mathrm{CAD}$ is decomposed by Bivariate 2D-EMD into a finite and small number of modes i.e., it is the sparse and multiscale decomposition. Qualitatively, the spatial frequency of the velocity fluctuations decreases when the number of the mode increases: the higher the mode order, the larger the scales of the corresponding flow structures. This physical feature can be quantified by computing the power spectral density (PSD) of each BIMF. The longitudinal PSD of each velocity component and their corresponding modes are presented in Fig. 8. It should be mentioned that they are computed from the velocity fluctuations by using Welch's method and the velocity field inside cylinder clearance volume is not considered. As one can see the maximum energy of the different modes is almost concentrated in different wave numbers, which ensures the scale separation of the instantaneous velocity field. The slope of the reference line is $-5 / 3$ corresponding to the inertial-range Kolmogorov spectrum. The first mode with the highest frequency and the lowest level of energy can be partially associated with the incoherent measured noise or the smallest resolvable scales within PIV data. 


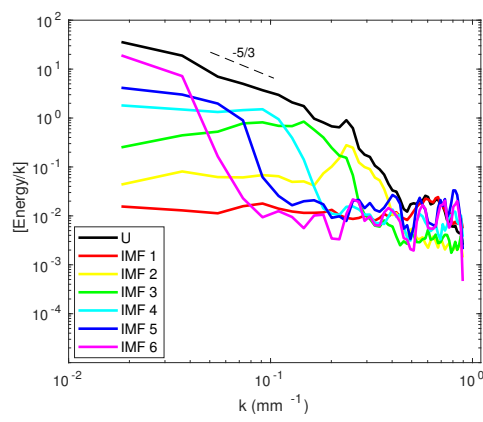

(a)



(b)

Fig. 8: The longitudinal PSD of each components of the velocity field and their corresponding modes, (a) Horizontal, $U$ and (b) Vertical, $V$ component at $100 \mathrm{CAD}$

The intermediate modes, modes 2-5, are mainly associated with the turbulence inertial sub-range and the last mode corresponds to the large-scale structures of the flow i.e., organized motion. These structures that contain the large amount of in-cylinder flow kinetic energy can be separated from turbulent fluctuations by using an energy criterion defined as

$$
E_{n}=0.5\left(\overline{U_{n}^{2}}+\overline{V_{n}^{2}}\right),
$$

Where $n$ denotes the mode number and the symbol - is the spatial average. This criterion is computed for the aforementioned velocity field at $100 \mathrm{CAD}$ and the result is presented in Fig. 9. A sudden increase in the energy of the last mode is observed, it is considered as a large-scale organized motion (flow Lf part). Meanwhile, the sum of the first five modes represents the turbulent fluctuations (flow Hf part), as shown in Fig.10.

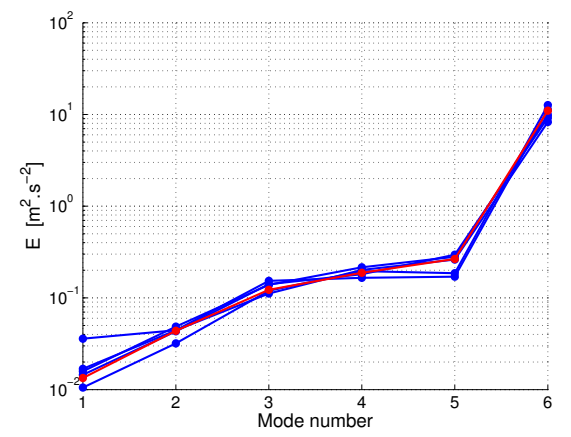

Fig. 9: Energy content as a function of mode numbers for decomposed velocity field at $100 \mathrm{CAD}$; the red line corresponds to the velocity field presented in Fig. $6 \mathrm{a}$ and the blue lines correspond to the velocity fields in the same CAD for 5 different cycles 


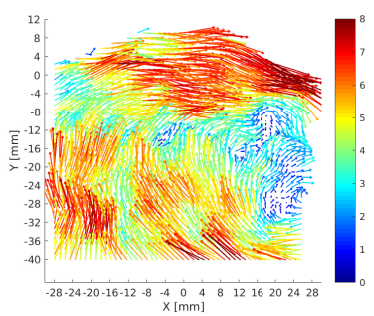

(a)

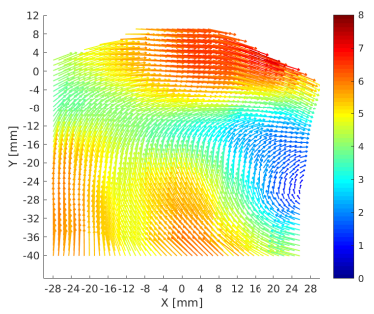

(b)

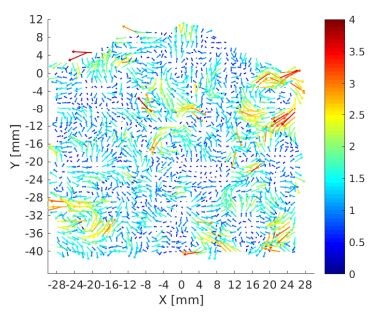

(c)

Fig. 10: (a) In-cylinder flow velocity field at 100 CAD (b) Low frequency part of the velocity field that considered as a large-scale organized motion, (c) High frequency part that represents the turbulent fluctuations. The unit is $\mathrm{ms}^{-1}$ and every two vectors are displayed

In this study, the analysis of the velocity fields is performed over 15 individual cycles between $100 \mathrm{CAD}$ to TDC during the compression stroke, and the same behavior was observed but not shown here. Indeed in all decomposed velocity fields a sudden increase in the last mode was observed. Also for illustration purpose, the instantaneous velocity fields at three different CADs, the corresponding Lf and Hf parts, the cyclic fluctuations of the Lf part of the flow $\left(\mathbf{U}_{\mathbf{c}}\right)$ and the phase-averaged velocity fields, are presented in Fig. 11. It is observed that the global feature of the flow (i.e., the large-scale organized motion) is quite well identified in the Lf part of the flow, presented in second row of Fig. 11. There is a significant similarity between the velocity field of this flow and the phase-averaged velocity field, shown in third row, however the flow instantaneous large-scale structure of considered cycle is better captured in Lf part, particularly in 60 and $30 \mathrm{CAD}$.

In the present study, Bivariate 2D-EMD is treated in parallel using Matlab_R2018b and performed on the IFPEN supercomputer (Intel Skylake G6140 processors). The decomposition of one velocity field requires about 54 CPU hours which represents an elapsed real time of 1.5 hours over 36 cores. 

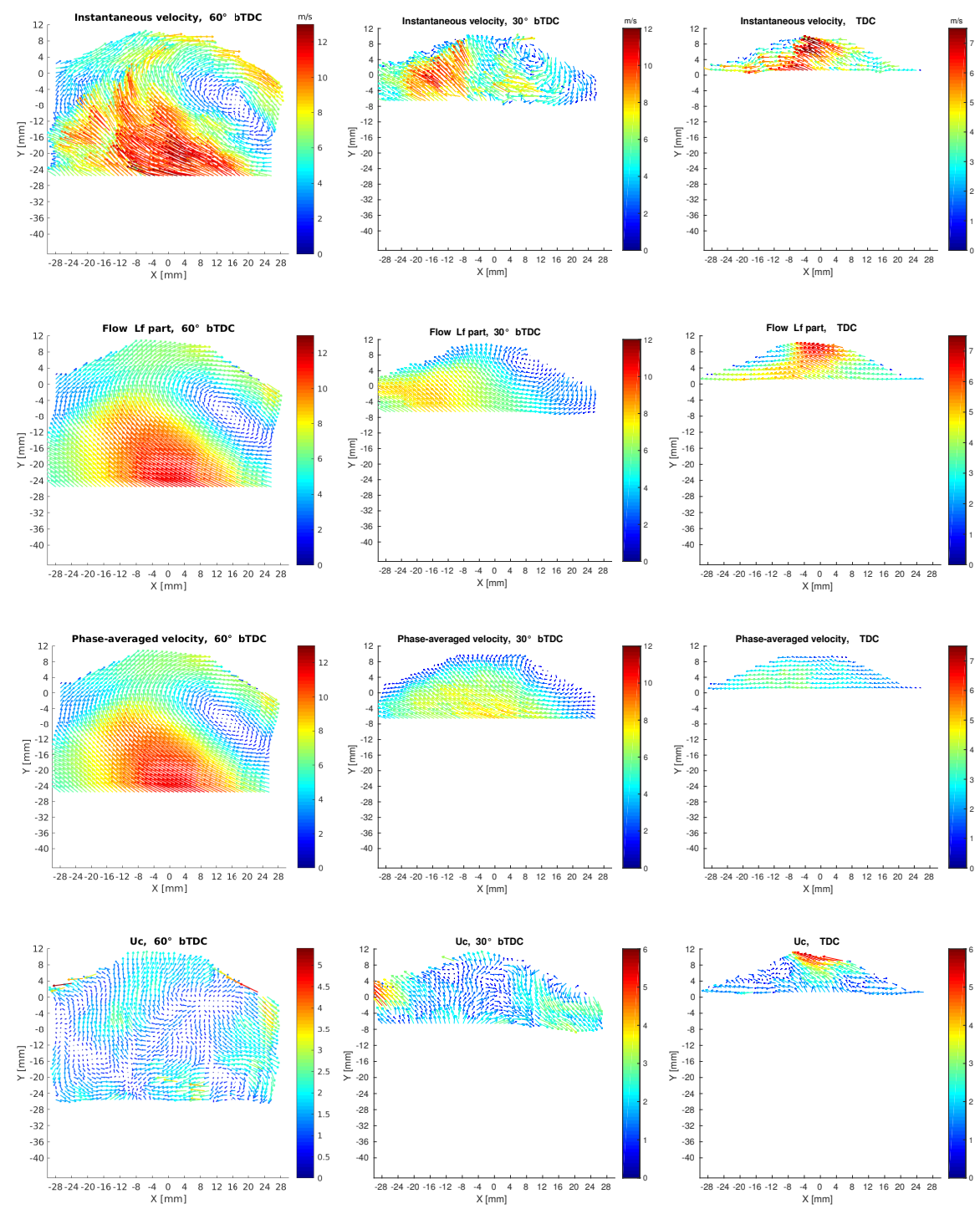

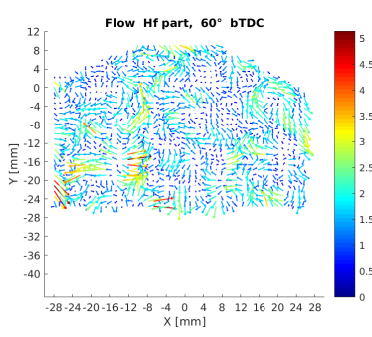

(a)

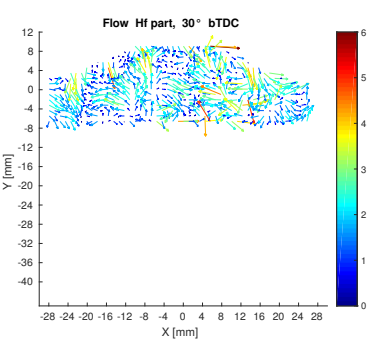

(b)

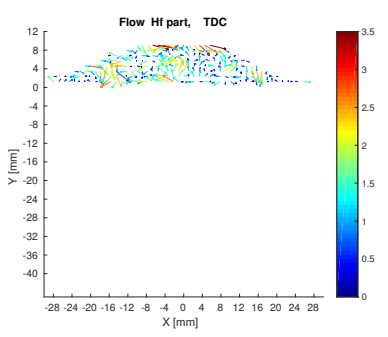

(c)

Fig. 11: Decomposition of instantaneous in-cylinder velocity; (a-c) 60 CAD, 30 CAD and TDC, respectively. First row: Instantaneous velocity, Second row: Lf part of the velocity, Third row: Phase-averaged velocity, Fourth row: $\mathbf{U}_{\mathbf{c}}$ and Fifth row: Hf part of the velocity field. The unit is $\mathrm{ms}^{-1}$ and every two vectors are depicted 
3.3 Estimation of in-cylinder turbulent length-scales

In order to show a benefit of such decomposition, the turbulent integral lengthscale for the Lf and the Hf parts of the flow is compared together. At a given time, spatial correlation coefficients (two points-one time) for $2 \mathrm{D}$ velocity field is defined as

$$
R_{i i}^{k}(\xi)=\frac{\left\langle u_{i}^{\prime}\left(x_{k}\right) \cdot u_{i}^{\prime}\left(x_{k}+\xi\right)\right\rangle}{\left\langle u_{i}^{\prime 2}\left(x_{k}\right)\right\rangle^{1 / 2} \cdot\left\langle u_{i}^{\prime 2}\left(x_{k}+\xi\right)\right\rangle^{1 / 2}},
$$

where $i=1,2$ denotes the velocity component, there is no Einstein summation over index, and $k=1,2$ indicates the coordinate direction. The $\xi$ is the spatial shift, $\langle$.$\rangle denotes spatial average and u_{i}^{\prime}$ is the velocity fluctuations computed from all the values in a velocity map. The integral length-scale is defined as $L_{i i}^{k}=\int_{0}^{\infty} R_{i i}^{k}(\xi) . d \xi[35]$. The longitudinal $\left(R_{U x}, R_{V y}\right)$ and lateral $\left(R_{U y}, R_{V x}\right)$ correlation curves and the corresponding integral length-scales $\left(L_{U x}, L_{V y}\right.$ and $L_{U y}, L_{V x}$, respectively) for the instantaneous velocity field and its different parts are presented in Fig. 12.

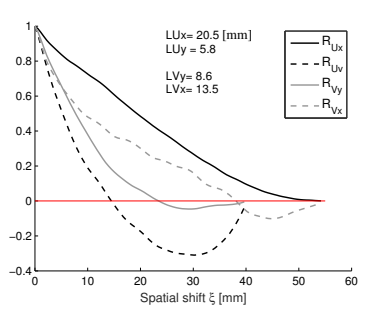

(a) $\mathbf{U}$

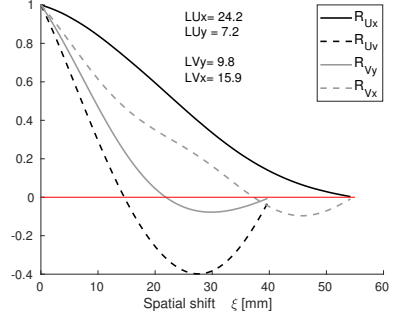

(b) $\mathbf{U}_{\mathbf{L f}}$

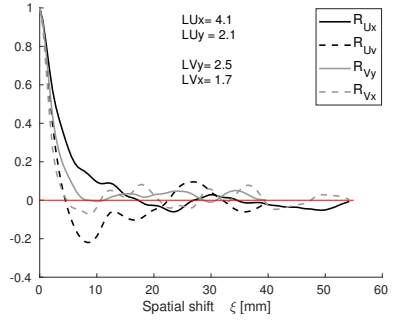

(c) $\mathbf{U}_{\mathbf{H f}}$

Fig. 12: Profile of the longitudinal and lateral correlation coefficients and the corresponding integral length-scale, in mm. (a) Instantaneous velocity field, (b) Lf part of the velocity, (c) Hf part of the velocity, at $100 \mathrm{CAD}$

It has to be mentioned that for computing correlation coefficients in Eq. (4), the velocity field above the clearance height (i.e., within the pentroof area) is not considered. Moreover the integral length scales are obtained by integrating spatial correlation functions until the first zero crossing, as presented in [25]. It can be observed that the evolution of the correlation curves obtained in the instantaneous flow field and its Lf part are quite close, but are smoother in the latter. However, the correlation curves associated with the Hf part of the flow are significantly different and the order of the integral length scales are smaller than that of Lf part. They represent the size of the turbulent eddies that are more pertinent for small scale mixing. These small structures of the flow are observed in Fig. 10c.

It is interesting to point out that in the Hf part of the flow, the longitudinal integral length-scale for each component of the velocity field $\left(L_{U x}, L_{V y}\right)$, is approximately two times the lateral ones $\left(L_{U y}, L_{V x}\right)$. In addition the lateral 
correlation curves of the two velocity components $\left(R_{U y}, R_{V x}\right)$ match closely. This singular feature of the high frequency flow is similar to that of homogeneous isotropic turbulence, although there is a difference between longitudinal correlation curves $\left(R_{U x}, R_{V y}\right)$. This analysis is also performed for the velocity fields at 70, 50 and $40 \mathrm{CAD}$, the obtained results are presented in Fig. 13. It is observed that the length scale associated with turbulent fluctuations $\left(\mathbf{U}_{\mathbf{H f}}\right)$ are quite distinct from that of the large-scale motion $\left(\mathbf{U}_{\mathbf{L f}}\right)$ and when the piston approaches to TDC, the scales of latter reduce.
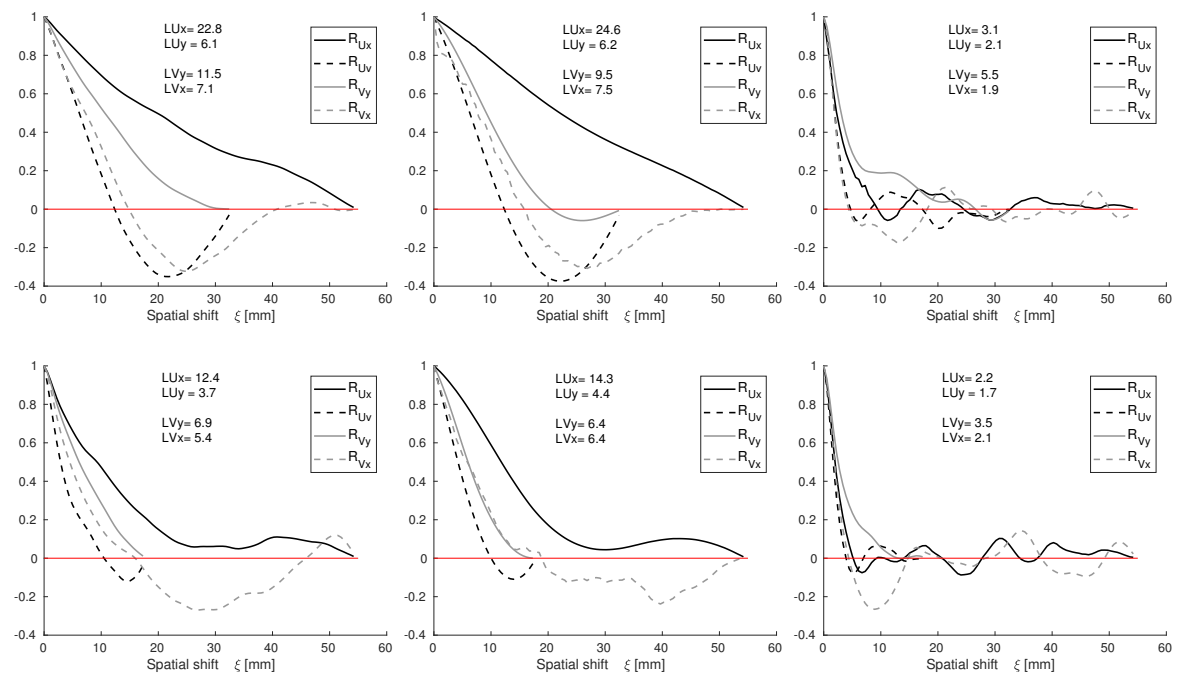

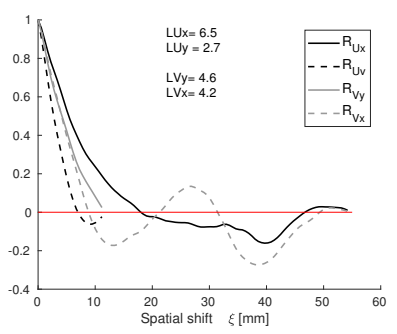

(a) $\mathbf{U}$

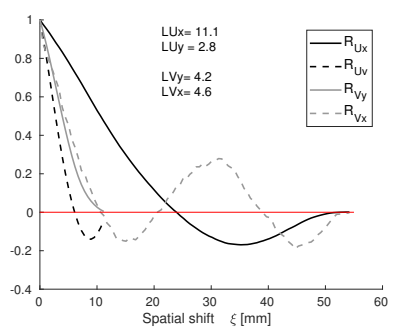

(b) $\mathbf{U}_{\mathbf{L f}}$

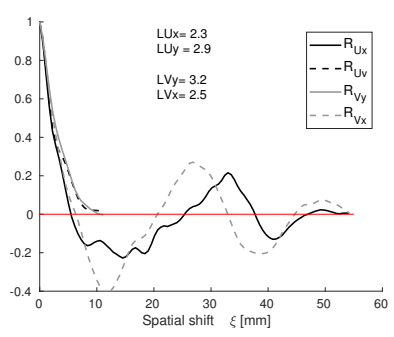

(c) $\mathbf{U}_{\mathbf{H f}}$

Fig. 13: Profile of the longitudinal and lateral correlation coefficients and the corresponding integral length-scale, in $\mathrm{mm}$. (a) Instantaneous velocity field, (b) Lf part of the velocity, (c) Hf part of the velocity. First row: 70 CAD, Second row: $50 \mathrm{CAD}$, Third row: $40 \mathrm{CAD}$ 


\section{In-cycle evolution of kinetic energy}

In this section, we present the use of Bivariate 2D-EMD as a non-statistical tool in order to estimate the contribution of turbulent kinetic energy and kinetic energy of cyclic fluctuations to the overall in-cylinder kinetic energy. The following analysis focuses on the second half of the compression stroke from $100 \mathrm{CAD}$ to TDC. At each crank angle degree, the instantaneous velocity field is decomposed by Bivariate 2D-EMD into different modes. The kinetic energy of each mode is computed in the same manner as the flow kinetic energy, following Eq. (3). In what follows, the crank angle evolution of the energy contained in each mode is presented in logarithmic scale and compared with the kinetic energy of the instantaneous flow field, see Fig. 14. As one can see, the energy levels of all modes are smaller than that of the instantaneous velocity of the flow and the evolution of the energy of the 6 -th mode is quite close to that of the instantaneous flow field along all CAD.



(a)

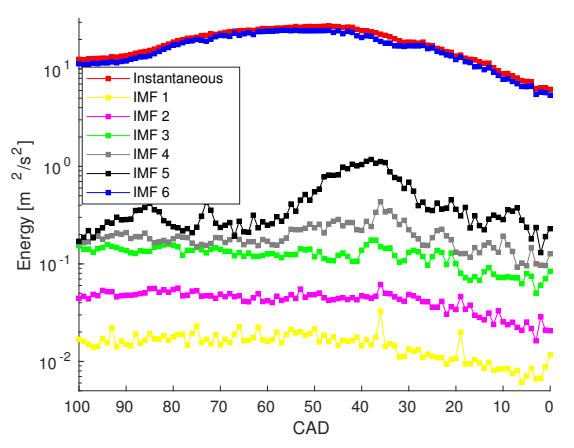

(b)

Fig. 14: The kinetic energy profile evolution of the different modes for two different cycles in (a) and (b)

The in-cylinder instantaneous velocity field can also be decomposed as

$$
\mathbf{U}=\langle\mathbf{U}\rangle+\mathbf{u},
$$

Where $\mathbf{U}$ is the instantaneous velocity with the components $(U, V, W)$ in $x$, $y$ and $z$ directions, respectively and $\mathbf{u}$ is the fluctuating velocity with the $(u, v, w)$ components. The symbol $\langle$.$\rangle denotes the phase-averaged operator.$ The PIV data used in this study only resolves $2 \mathrm{D}$ velocity field $(U, V)$ in the central symmetry plane with an area of $S$. The transverse velocity component $W$ is not available although it has been measured using tomographic PIV in the same engine and at the same operating conditions [2,52]. Such measurements are not used here because they only captured a single $3 \mathrm{D}$ velocity field per cycle, while the high-speed planar PIV provides velocity data at each crank-angle degree through the engine cycle. Therefore in this analysis, the $W$ 
velocity component and its fluctuations $w$ are not considered. The reader is referred to $[2,52]$ for further information about this component. Finally, the total phase-averaged kinetic energy per unit mass can be considered as a sum of the mean flow kinetic energy, $E_{m}$ and the phase-averaged fluctuating kinetic energy, $E_{f}$, as follows $[14,46]$ :

$$
E_{m}=\frac{1}{2 S} \int_{S}\left(\langle U\rangle^{2}+\langle V\rangle^{2}\right) d S, \quad E_{f}=\frac{1}{2 S} \int_{S}\left(\left\langle u^{2}\right\rangle+\left\langle v^{2}\right\rangle\right) d S .
$$

Considering this statistical approach for decomposition of the velocity field, $E_{f}$ includes the energy of both cycle-to-cycle fluctuations of the mean flow (i.e., the contribution of the in-cycle coherent motion) and the turbulent fluctuations carried by the fluid motion. The evolution of $E_{m}$ and $E_{f}$ for the studied in-cylinder flow is presented in Fig. 15a. Beyond about 50 CAD, a clear decrease in $E_{m}$ is observed. This decrease can be due to the reduction of the piston speed or as more likely due to the breakdown of the large-scale organized motion i.e., tumble flow to the turbulence smaller-scale. Nevertheless there is no significant increase in fluctuating kinetic energy, $E_{f}$, in this range as what was observed in [46]. Also the evolution of the instantaneous fluctuating kinetic energy described as $E_{i}=\frac{1}{2 S} \int_{S}\left(u^{2}+v^{2}\right) d S$, for three arbitrary cycles are presented in Fig.15b. Significant differences between them are observed, particularly for cycle 1 where the maximum value of the fluctuating kinetic energy is about twice that of the other two cycles. This kinetic energy contains both the contribution of turbulence and cyclic variability $(\mathrm{CCV})$ of the large-scale organized motion.

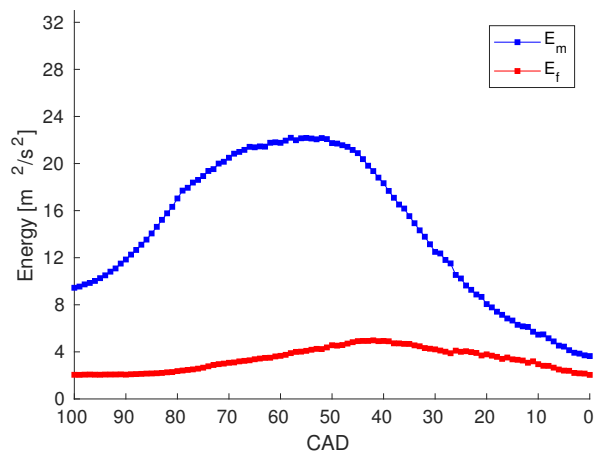

(a)

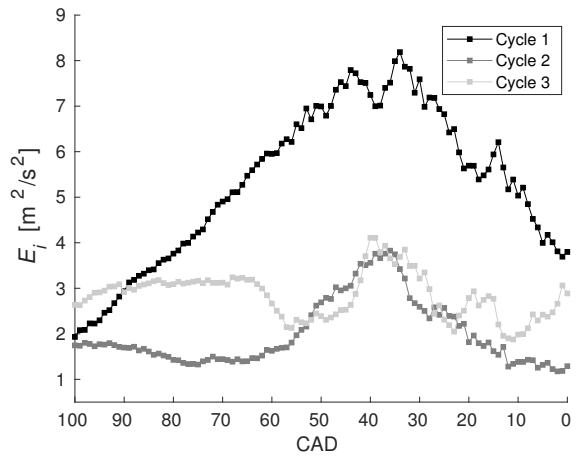

(b)

Fig. 15: (a) Evolution of the mean flow kinetic energy, $E_{m}$ and the phaseaveraged fluctuating kinetic energy, $E_{f}$ in Eq. (6), (b) Evolution of the instantaneous fluctuating kinetic energy, $E_{i}$ for three cycles

To shed light on this issue, the instantaneous velocity fields of these cycles are decomposed by Bivariate 2D-EMD and the large-scale structure of the flow (Lf part) is separated from the turbulent fluctuations (Hf part) for each 
velocity field. The kinetic energy of the instantaneous velocity field and the corresponding flow Lf and Hf part are compared in Fig.16.
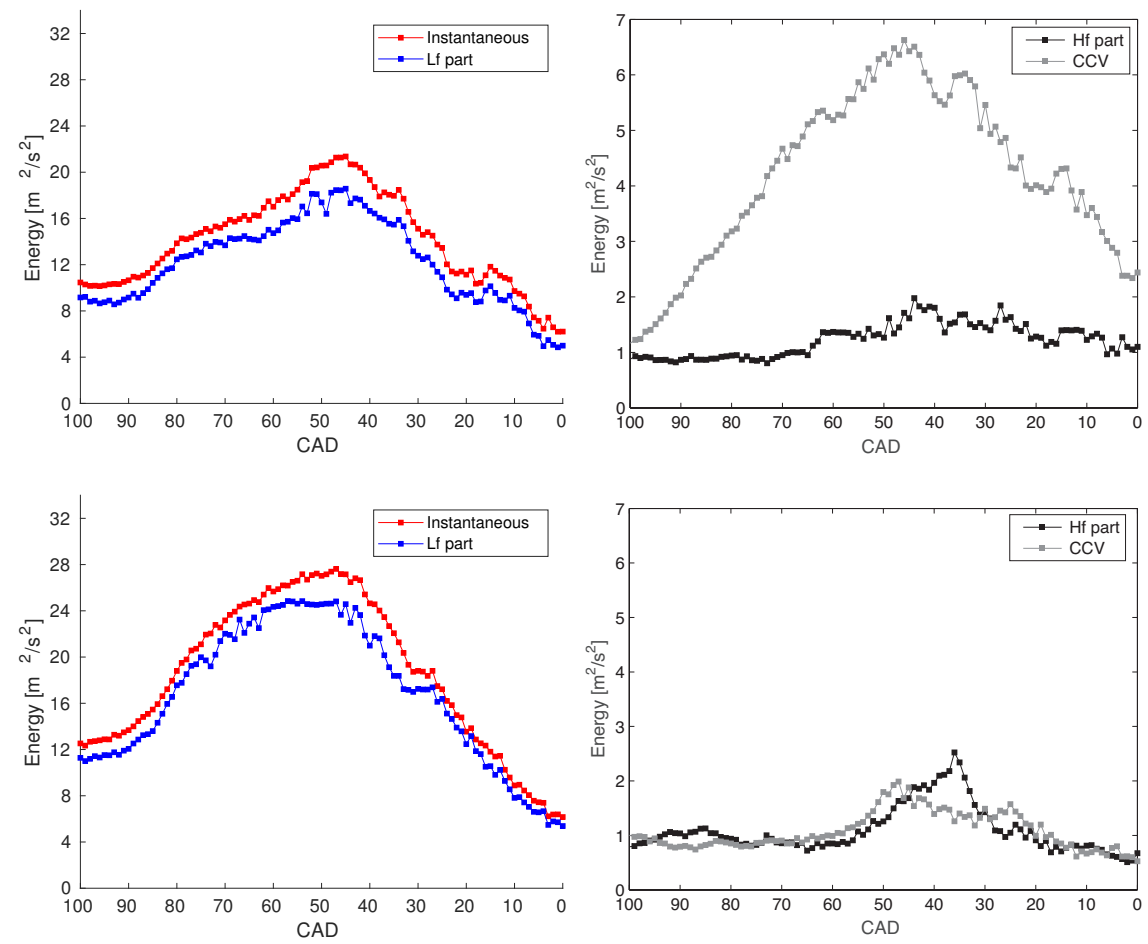

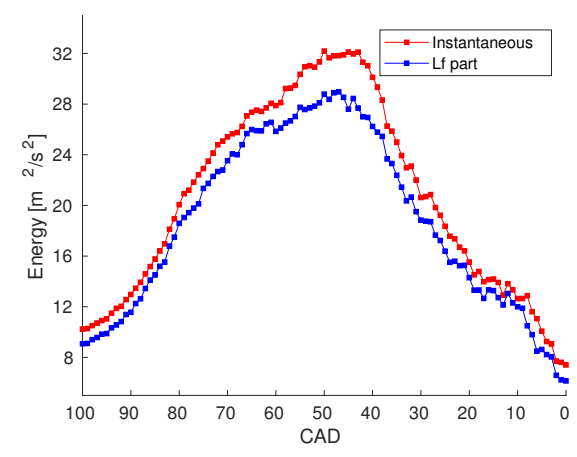

(a)

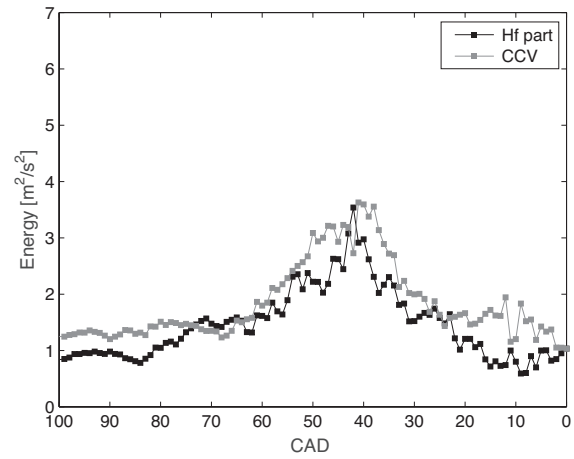

(b)

Fig. 16: Evolution of the kinetic energy for three arbitrary cycles; cycle 1, cycle 2 and cycle 3 from first to third row, respectively. (a) Instantaneous velocity field vs. Flow Lf part of EMD analysis, (b) Flow Hf part of EMD analysis vs. $\mathrm{CCV}$ that corresponds to $\mathbf{U}_{\mathbf{c}}=\mathbf{U}_{\mathbf{L f}}-\mathbf{U}_{\mathbf{E A}}$ 
As expected, the energy of the Lf part is smaller than the energy associated with the instantaneous flow. However, as shown in Fig. 16a, these curves exhibit the same energy behavior during the compression phase. It is also observed that the maximum kinetic energy of the instantaneous flow in cycle 1 is the smallest of the three cycles. The kinetic energy contributions of the Hf part, which represents the turbulence, and of the flow CCV are plotted in Fig. 16b. For the three considered cycles, their values are much lower than that of the Lf parts but very close to each other except for the cyclic variation of cycle 1 which maximum value is about 3 times higher than that of the two other cycles. Cycle 1 is characterized by large scale structures which presents smaller velocities and a singular behavior compared with the two other cycles. For illustration, the velocity fields of $\mathbf{U}_{\mathbf{c}}$ for the three cycles at $50 \mathrm{CAD}$ are compared in Fig. 17. It is observed that the magnitude of the velocity cyclic variation reaches higher values (by at least a factor 2) in a large region located under the intake valves.



(a) Cycle 1

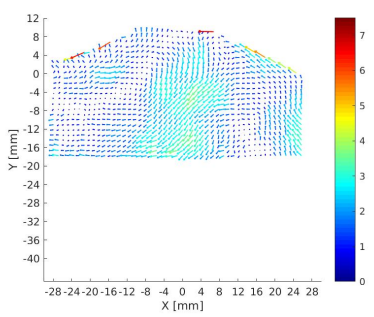

(b) Cycle 2

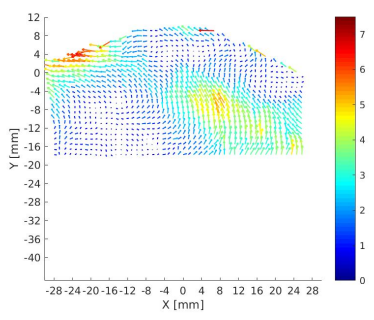

(c) Cycle 3

Fig. 17: Comparing the velocity field of $\mathbf{U}_{\mathbf{c}}$ for three different cycles at 50 CAD. The unit is $\mathrm{m} \mathrm{s}^{-1}$ and every two vectors are displayed

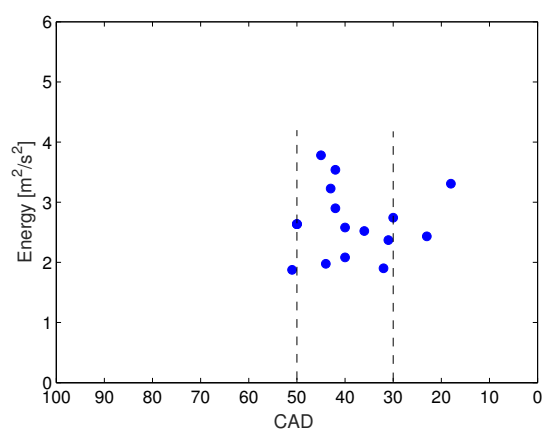

(a)

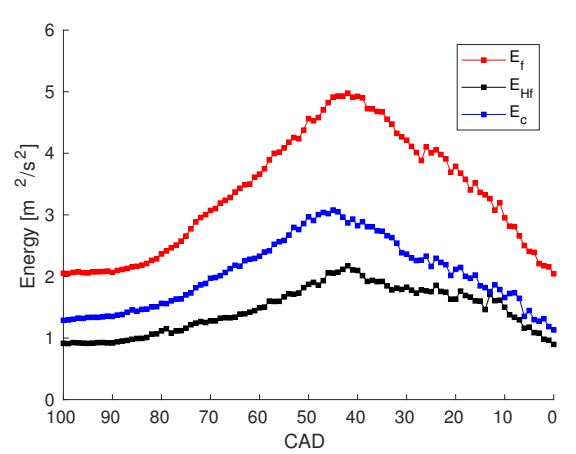

(b)

Fig. 18: (a) The maximum of the flow Hf part kinetic energy as a function of CAD for all cycles, (b) The contribution of flow Hf part i.e., turbulent fluctuations, Eq. (7) and cyclic variations in fluctuating kinetic energy, Eq. (8) 
Figure 18a represents the maximum of kinetic energy of $\mathbf{U}_{\mathbf{H f}}$ (i.e., turbulent kinetic energy) as a function of CAD for all analyzed cycles. For about $80 \%$ of the cycles, it is placed between 50 and 30 CAD. This energy promotes the flame kernel propagation hence such analysis should allow us to improve the phasing of the turbulence peak in order to get it closer to the ignition time.

The phase-averaged kinetic energy of Hf part of the flow, $E_{H f}$ and the one obtained from substraction of the phase-averaged velocity from the velocity of the Lf part, $E_{c}$ are defined as:

$$
\begin{gathered}
E_{H f}=\frac{1}{2 S} \int_{S}\left(\left\langle U_{H f}^{2}\right\rangle+\left\langle V_{H f}^{2}\right\rangle\right) d S \\
E_{c}=\frac{1}{2 S} \int_{S}\left(\left\langle\left(U_{L f}-\langle U\rangle\right)^{2}\right\rangle+\left\langle\left(V_{L f}-\langle V\rangle\right)^{2}\right\rangle\right) d S .
\end{gathered}
$$

$E_{c}$ corresponds to the kinetic energy of the Lf flow cyclic variability. Figure $18 \mathrm{~b}$ shows the contribution of these energies compared to the total fluctuating energy, $E_{f}$ in Eq. (6), in the second half of the compression stroke. The level of cyclic fluctuations is greater than that of the turbulent fluctuations and reaches about $66 \%$ of the total fluctuating energy at 45 CAD. Although afterwards this proportion decreases progressively, it still maintains a level of $55 \%$ near TDC.

\section{Tumble center trajectory analysis}

This section is dedicated to the interest of Bivariate 2D-EMD to help identifying the tumble center position and track its progression through the compression stroke. Tumble flow is the generic large-scale organized motions in the cylinder of spark-ignition engine. It is a bulk fluid motion about the axis perpendicular to the cylinder axis and to the symmetry plane. Tumble motion has two important roles. Firstly, it stores flow momentum and energy and give them back at the end of the compression phase in the form of turbulence with characteristics allowing a sufficiently fast propagation of the turbulent flame generated by the spark. Secondly, it can increase the mean flow energy by compression process, effectively generating more turbulent kinetic energy near TDC by tumble breakdown. The fluctuations of the tumble center position and its intensity highly affect the air-fuel mixing process and combustion development $[4,29]$. Commonly an Eulerian approach based on the flow field topology is used in order to identify the position of the tumble center [18]: $\Gamma(P)=\frac{1}{N} \sum_{S} \frac{\left(\overrightarrow{P M} \wedge \overrightarrow{U_{M}}\right) . z}{\|\overrightarrow{P M}\| \cdot\left\|\overrightarrow{U_{M}}\right\|}$, where $\Gamma$ is the dimensionless scalar function that is bounded by 1 and reaches a maximum value near the vortex core. $S$ is the two dimensional area, rectangular in the case of PIV datasets, centered on $P, N$ is the number of points $M$ inside $S$ and $z$ is the unit vector normal to the measurement plane. The operator $\|$.$\| represents the Euclidean norm$ of the vector. The tumble center position computed by this method is highly 
sensitive to the size of sub-region $S$, this effect is detailed hereafter.

Several studies were dedicated to the tracking of the tumble center trajectory during intake and compression stroke. However due to the in-cylinder flow complexity, the presence of local vortices with different sizes and the considerable amount of the flow turbulent fluctuations, the accurate identification of the fluid bulk motion is not straightforward. Druault et al. [11], studied the evolution of the tumble center in the most energetic modes of snapshots POD that resulted from 32 consecutive cycles and compared it with the ones obtained from phase-averaged and instantaneous velocity fields. Muller et al. [32], by applying POD on 73 cycles, studied the average location of the tumble center position during the second half of the compression stroke.

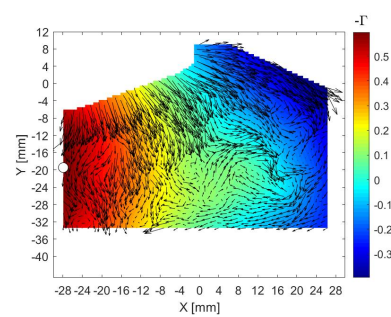

(a)

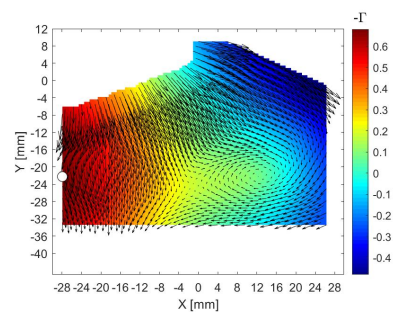

(d)

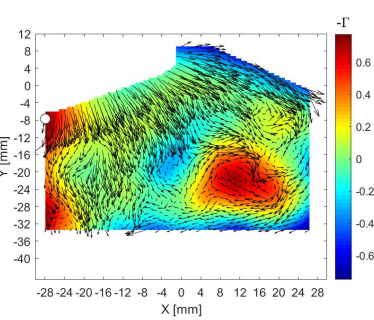

(b)

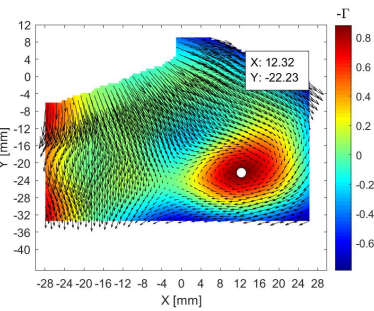

(e)

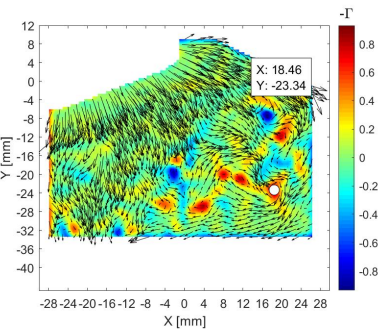

(c)

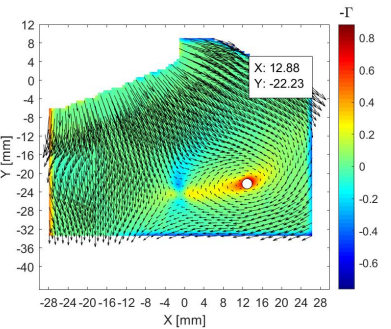

(f)

Fig. 19: Identification of the tumble center position at 290 CAD. First row: Instantaneous velocity field, Second row: Flow Lf part of the EMD analysis. The function $\Gamma(P)$ is computed by considering the entire domain (a and d), a quarter of the domain (b and e) and $3 \times 3$ grid points (c and f) as a $S$ sub-region. Due to the flow clockwise rotation, its value is negative at tumble center. Every four vectors are displayed

However regarding EMD analysis, there is no need to have several resolved cycles for analyzing tumble motion dynamics. Indeed the instantaneous velocity fields in individual cycle are decomposed by Bivariate 2D-EMD and the turbulent fluctuations are separated from large-scale organized motion, so the position of the tumble center is computed in the latter. For illustration purposes, the position of the tumble center for a given velocity field in intake 
stroke at $290 \mathrm{CAD}$ is computed for both the instantaneous velocity field and the low frequency part of the EMD analysis. The results are compared in the first and second rows of Fig. 19. In addition, the different sizes of $S$ area for computing the $\Gamma(P)$ function are investigated using either the entire domain (Figs. 19a and 19d), or a quarter of the velocity field (Figs. 19b and 19e) or a $3 \times 3$ grid points (Figs. 19c and 19f). Since the turbulent fluctuations have been removed in the Lf part, the tumble center position can be precisely identified in cases where a quarter of the velocity field and $3 \times 3$ grid points are considered.

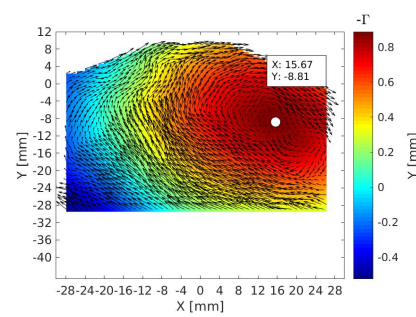

(a)

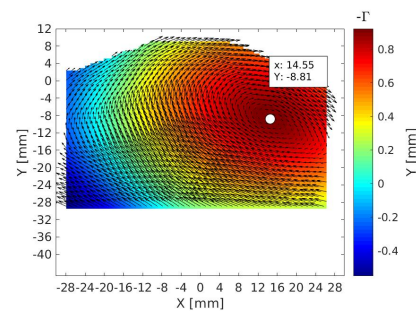

(d)

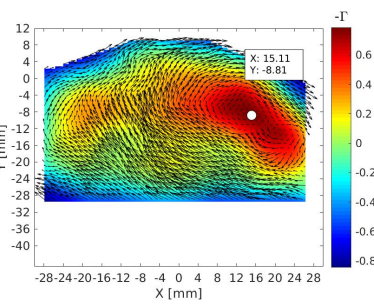

(b)



(e)

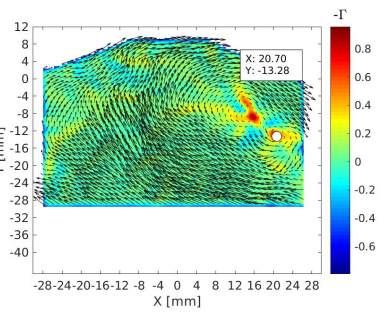

(c)

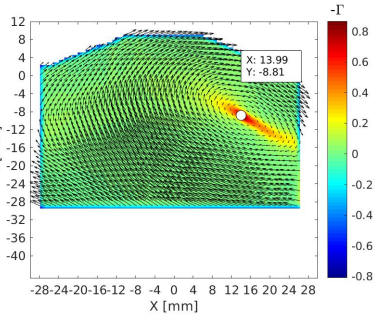

(f)



(g)

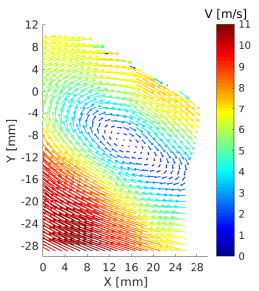

(h)

Fig. 20: Identification of the tumble center position at 65 CAD. First row: Instantaneous velocity field, Second row: Flow Lf part of the EMD analysis. The function $\Gamma(P)$ is computed by considering the entire domain (a and d), a quarter of the domain (b and e) and $3 \times 3$ grid points (c and f) as a $S$ sub-region. Due to the flow clockwise rotation, its value is negative at tumble center. Every four vectors are displayed. Third row: Zoom region near tumble core on instantaneous velocity field $(\mathrm{g})$ and Lf part of the flow from EMD analysis (h), every two vectors are displayed 
Another example in which the tumble center position is identified in the compression stroke at $65 \mathrm{CAD}$, is illustrated in Figs. 20. The results are consistent with those of previous case. Also the presence of two local vortices in the instantaneous velocity field compared with the global rotational motion (tumble vortex) center are shown in Fig. 20g and Fig. 20h, respectively.

For a given cycle, the tumble center footprint is identified by considering $3 \times 3$ grid points as a $S$ sub-region. This procedure is performed for both the instantaneous velocity field and the Lf field. The results are compared in Fig. 21 , a significant difference between two trajectories is observed.

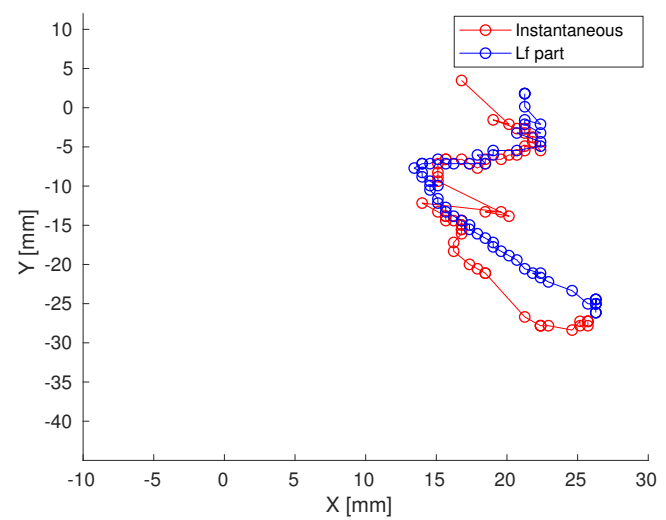

Fig. 21: Identification of the tumble center trajectory during compression stroke from 100 to $40 \mathrm{CAD}$ in instantaneous velocity field v.s. flow Lf part of EMD analysis

\section{Conclusions}

This study proposes Bivariate 2D-EMD for analyzing the instantaneous incylinder flow velocity in order to separate turbulent fluctuations from flow large-scale organized structures. The interest of such distinction is to estimate turbulent intensity which plays a significant role on combustion development and also to provide a quantitative analysis of CCV in engines. Contrary to statistical methods, this approach is appropriate for analyzing unsteady turbulent in-cylinder flows and to capture crank-angle resolved large-scale structure evolution within individual cycle. The fundamental point is that in this methodology there is no need to have numerous velocity fields at a given crank angle. Single velocity field is decomposed into several modes then, based on energy consideration, the turbulent fluctuations i.e., the high frequency part of the flow (Hf) are separated from the mean flow i.e., the low frequency part of the flow (Lf).

Consequently, an advantage of EMD analysis is that the large-scale organized 
motion appears in the last mode of the decomposition. This implies that EMD analysis does not require a formal criterion such as a cut-off frequency or to truncate the series of the modes to distinguish the large scale structures of the flow from the turbulence which makes this approach more adapted to analyze the whole engine cycle without any adjustment. The methodology is applied to a high-speed planar PIV data set performed in an optically accessible engine. The measurement plane is restricted to the central symmetry plane and contains the clearance volume. The analysis focuses on the second half of the compression stroke, $100 \mathrm{CAD}$ to TDC. The velocity fields from 15 individual cycles are analyzed and the contribution of the turbulent fluctuations and $\mathrm{CCV}$ in fluctuating kinetic energy is investigated. Furthermore, the estimation of the flow length scales associated with turbulent fluctuations and the large-scale motion is performed. Finally, the benefit of such approach to accurately identify the tumble center trajectory is demonstrated. The proposed approach appears to be a promising methodology to analyze instantaneous in-cylinder velocity fields measured experimentally or resolved by numerical simulations. In addition, when engine operates under speed transient conditions, the simulation of adequate cycles to perform statistical analysis has a huge computational cost. The EMD analysis of in-cylinder velocity fields obtained by LES is the subject of ongoing work for spray-guided engine in motored and reactive conditions.

Conflict of interest The authors declare that they have no competing interests.

Acknowledgments The authors would like to express their gratitude to Pr. Jacques Borée (Institut Pprime, CNRS, Univ. Poitiers - ENSMA), Pr. Karim Abed-Meraim (Prisme Laboratory, Univ. Orléans) and Dr. Laurent Duval (IFPEN, Rueil-Malmaison) for enriching discussions.

Benjamin Böhm kindly acknowledges generous support by Deutsche Forschungsgemeinschaft through FOR 2687 "Cyclic variations in highly optimized sparkignition engines: experiment and simulation of a multi-scale causal chain" project number 423224402. Brian Peterson kindly acknowledges financial support by the European Research Council (ERC, grant no. 759546). 


\section{Appendix}

A synthetic Lamb-Oseen vortex, $\mathrm{U}_{i}(x, y)$ that mimics large-scale organized motion is perturbed by an experimental HIT velocity field, $\mathrm{P}(x, y)$ within $127 \times 127$ data points with spatial resolution of $0.16 \mathrm{~mm}$ [16], shown in Fig. $22 \mathrm{a}$ and $22 \mathrm{~b}$, respectively. The HIT flow has a longitudinal integral lengthscale of $3.2 \mathrm{~mm}$ and the scale of vortex is approximately five times larger. The primary perturbed velocity field in Fig. 22c is interpolated on $254 \times 254$ data points with spatial resolution of $0.08 \mathrm{~mm}$, as presented in Fig. 22d.

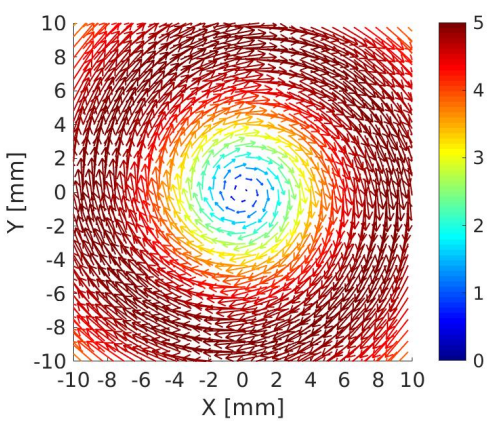

(a)

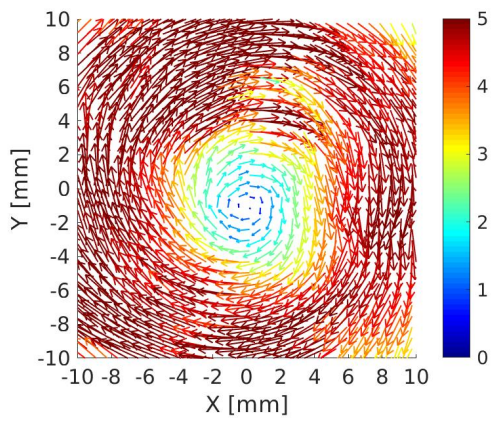

(c)

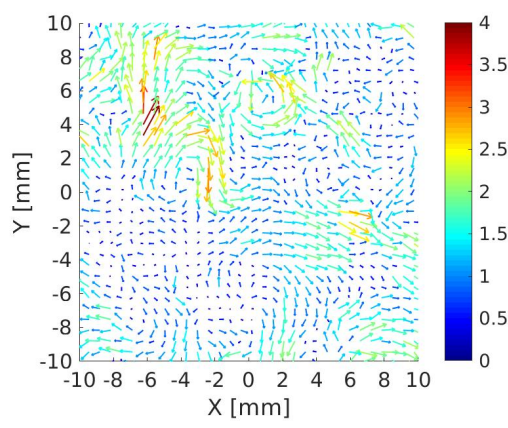

(b)



(d)

Fig. 22: (a) Large-scale synthetic flow, (b) Experimental HIT velocity field as a perturbation, (c-d) Perturbed velocity field within $127 \times 127$ and $254 \times 254$ data points, respectively. Every four vectors are displayed, the unit is $\mathrm{ms}^{-1}$

The primary and interpolated velocity fields are decomposed by Bivariate 2D-EMD. The modes corresponding to the horizontal velocity components, $U$ are shown in Fig. 23. As one can see the interpolated velocity field has one mode more than the primary field, however the last modes of both fields are quite similar. Also the 5 -th mode of the primary perturbed velocity field corresponds to the 6 -th mode of the interpolated one and so on up to the first mode of the primary field that is distributed on the first and second modes of the interpolated velocity field. 
Title Suppressed Due to Excessive Length

29
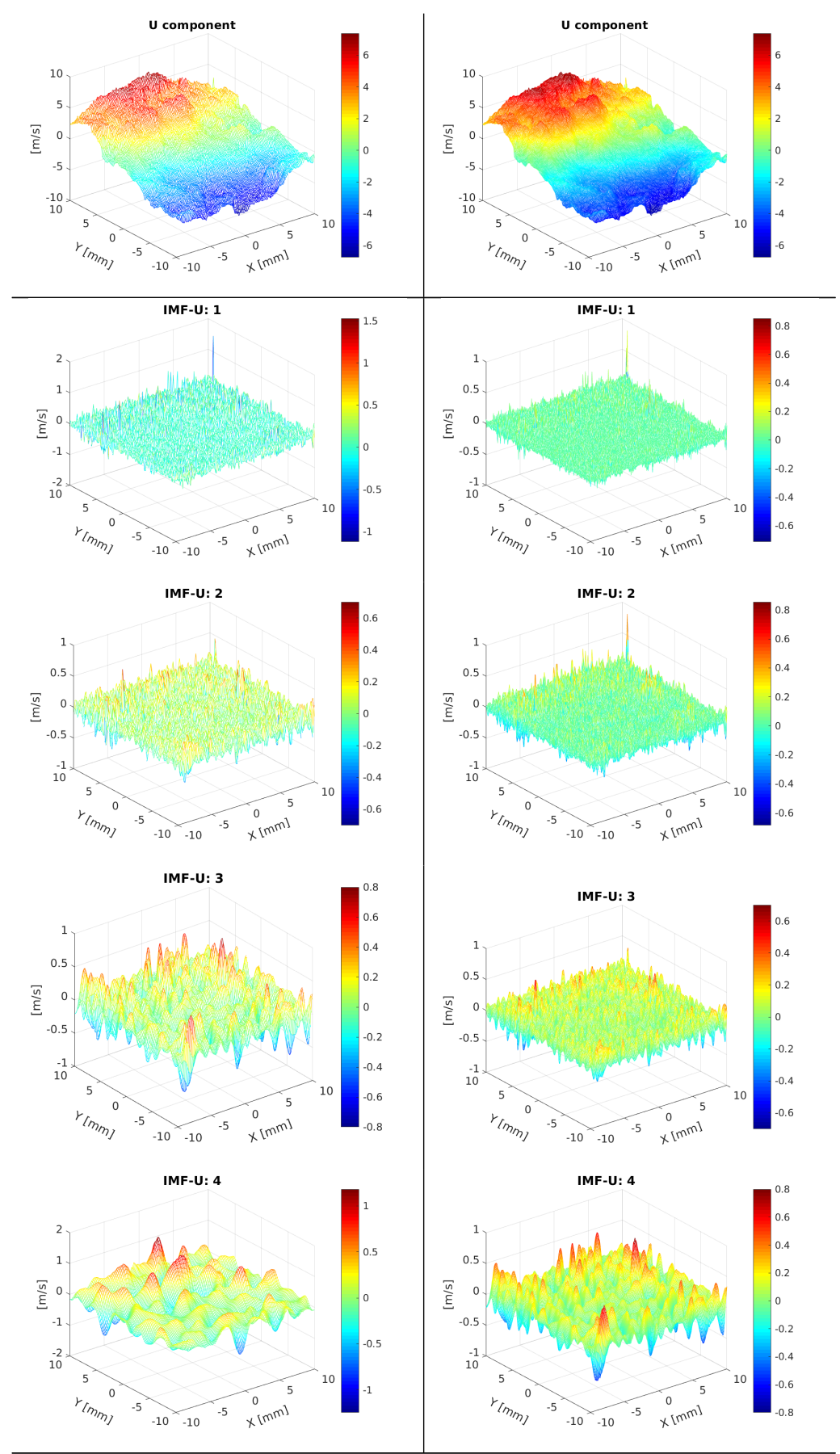

Fig.23, Cont. 

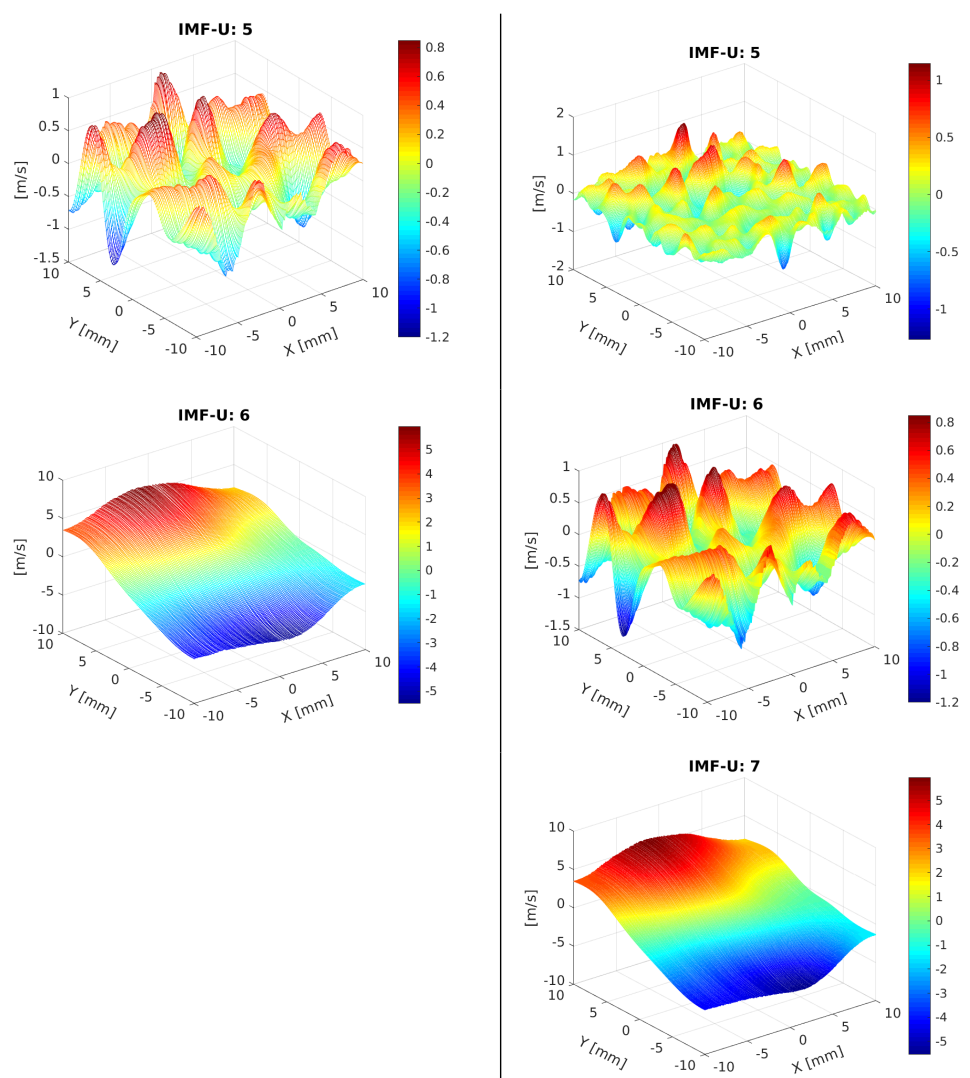

(a)

(b)

Fig. 23: Horizontal velocity field in the first row and the corresponding modes in following. (a) Primary, (b) Interpolated velocity fields, the unit is $\mathrm{m} \mathrm{s}^{-1}$

Figure 24 presents the energy content of each mode for two decomposed velocity fields in logarithmic scale. For both cases, a significant increase in the energy of last mode is observed. This mode is considered as the organized motion $\left(\mathbf{U}_{\mathbf{L f}}\right)$ and the sum of the other modes represents the HIT velocity field $\left(\mathbf{U}_{\mathbf{H f}}\right)$. These flow fields are illustrated in the first and second row of Fig. 25 for the primary and interpolated velocity field, respectively. By observation, there is a good agreement between the flow structures of two Hf velocity fields as well as two Lf ones. Moreover the relative mean square error between mean kinetic energy of the Hf velocity fields is $6.7 \%$ and that of the Lf velocity fields is $0.3 \%$.

As a conclusion, the interpolation of the velocity field on the grid points with higher spatial resolution affects slightly the feature of the low order modes that contain highest spatial frequency. Indeed the first mode of the primary perturbed velocity field, that can be considered as a measurement error or 
incoherent noise, is distributed on the first and second modes of the interpolated velocity field. However, it has no influence on the higher order modes, in particular the last one that represents the flow large-scale organized motion i.e., Lf part of the flow.

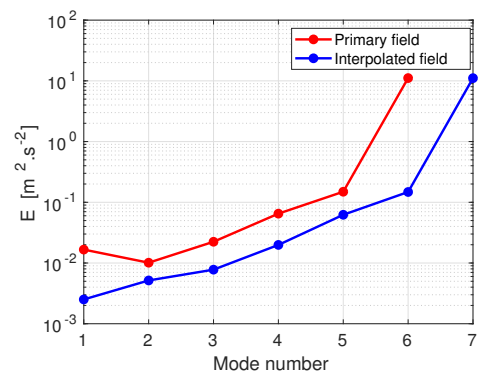

Fig. 24: Energy content of each mode for two decomposed perturbed velocity fields

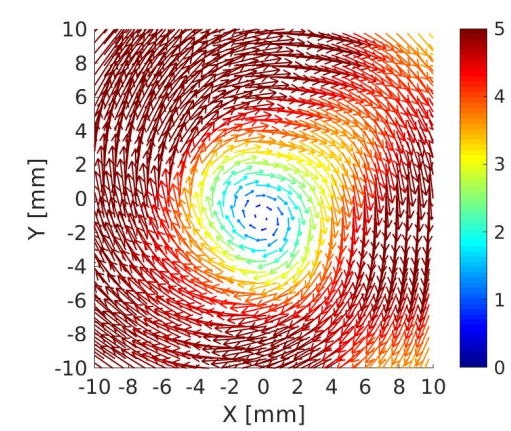

(a)

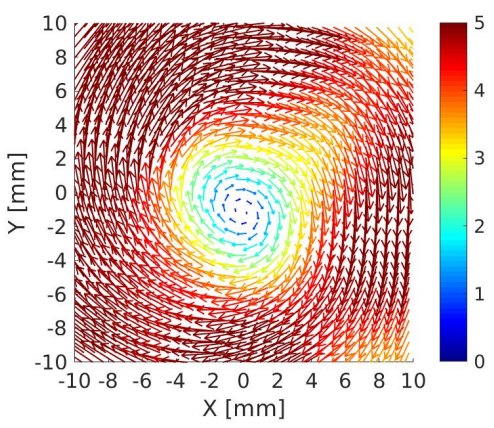

(c)



(b)



(d)

Fig. 25: (a-b) Flow Lf and Hf velocity field, respectively correspond to the primary perturbed velocity field. Every four vectors are displayed, (c-d) Flow Lf and Hf velocity field, respectively correspond to the interpolated perturbed velocity field. Every eight vectors are displayed, the unit is $\mathrm{ms}^{-1}$ 


\section{References}

1. Abraham, P., Reuss, D., Sick, V.: High-speed particle image velocimetry study of incylinder flows with improved dynamic range. Tech. rep., SAE Technical Paper (2013)

2. Baum, E., Peterson, B., Böhm, B., Dreizler, A.: On the validation of LES applied to internal combustion engine flows: part 1: comprehensive experimental database. Flow, turbulence and combustion 92(1-2), 269-297 (2014)

3. Bizon, K., Continillo, G., Lombardi, S., Sementa, P., Vaglieco, B.M.: Independent component analysis of cycle resolved combustion images from a spark ignition optical engine. Combustion and Flame 163, 258-269 (2016)

4. Borée, J., Miles, P.C.: In-Cylinder Flow. Encyclopedia of automotive engineering pp. $1-31(2014)$

5. Cao, J., Ma, Z., Li, X., Xu, M.: Three dimensional proper orthogonal decomposition analysis of engine in-cylinder velocity fields. Measurement Science and Technology (2019)

6. Cao, Y., Kaiser, E., Borée, J., Noack, B.R., Thomas, L., Guilain, S.: Cluster-based analysis of cycle-to-cycle variations: application to internal combustion engines. Experiments in fluids 55(11), 1837 (2014)

7. Chen, H., Reuss, D.L., Sick, V.: On the use and interpretation of proper orthogonal decomposition of in-cylinder engine flows. Measurement Science and Technology 23(8), $085302(2012)$

8. Clark, L.G., Kook, S.: Correlation of spatial and temporal filtering methods for turbulence quantification in spark-ignition direct-injection (sidi) engine flows. Flow, Turbulence and Combustion 101(1), 161-189 (2018)

9. Cosadia, I., Borée, J., Charnay, G., Dumont, P.: Cyclic variations of the swirling flow in a Diesel transparent engine. Experiments in fluids 41(1), 115 (2006)

10. Dragomiretskiy, K., Zosso, D.: Variational mode decomposition. IEEE transactions on signal processing 62(3), 531-544 (2013)

11. Druault, P., Guibert, P., Alizon, F.: Use of proper orthogonal decomposition for time interpolation from PIV data. Experiments in fluids 39(6), 1009-1023 (2005)

12. Feynman, J., Ruzmaikin, A.: The Centennial Gleissberg Cycle and its association with extended minima. Journal of Geophysical Research: Space Physics 119(8), 6027-6041 (2014)

13. Fogleman, M., Lumley, J., Rempfer, D., Haworth, D.: Application of the proper orthogonal decomposition to datasets of internal combustion engine flows. Journal of Turbulence 5(23), 1-3 (2004)

14. Fogleman, M., Rempfer, D., Lumley, J.L., Haworth, D.: POD Analysis of In-Cylinder Flows. In: ASME 2002 Joint US-European Fluids Engineering Division Conference, pp. 1173-1178. American Society of Mechanical Engineers (2002)

15. Freudenhammer, D., Peterson, B., Ding, C.P., Boehm, B., Grundmann, S.: The influence of cylinder head geometry variations on the volumetric intake flow captured by magnetic resonance velocimetry. SAE International Journal of Engines 8(4), 1826-1836 (2015)

16. Galmiche, B., Mazellier, N., Halter, F., Foucher, F.: Turbulence characterization of a high-pressure high-temperature fan-stirred combustion vessel using ldv, piv and tr-piv measurements. Experiments in fluids 55(1), 1636 (2014)

17. Gosman, A.: Flow processes in cylinders. Thermodynamics and gas dynamics of internal combustion engines 2, 616-772 (1986)

18. Graftieaux, L., Michard, M., Grosjean, N.: Combining PIV, POD and vortex identification algorithms for the study of unsteady turbulent swirling flows. Measurement Science and technology 12(9), 1422 (2001)

19. Heim, D.M., Jesch, D., Ghandhi, J.B.: Size-scaling effect on the velocity field of an internal combustion engine, part ii: Turbulence characteristics. International Journal of Engine Research 15(2), 193-208 (2014)

20. Hemakom, A., Goverdovsky, V., Looney, D., Mandic, D.P.: Adaptive-projection intrinsically transformed multivariate empirical mode decomposition in cooperative braincomputer interface applications. Phil. Trans. R. Soc. A 374(2065), 20150199 (2016)

21. Heywood, J.B.: Internal combustion engine fundamentals (1988) 
22. Huang, N.E., Shen, Z., Long, S.R.: A new view of nonlinear water waves: the Hilbert spectrum. Annual review of fluid mechanics 31(1), 417-457 (1999)

23. Huang, N.E., Shen, Z., Long, S.R., Wu, M.C., Shih, H.H., Zheng, Q., Yen, N.C., Tung, C.C., Liu, H.H.: The empirical mode decomposition and the hilbert spectrum for nonlinear and non-stationary time series analysis. Proceedings of the Royal Society of London. Series A: mathematical, physical and engineering sciences 454(1971), 903-995 (1998)

24. Huang, Y.X., Schmitt, F.G., Lu, Z.M., Liu, Y.L.: An amplitude-frequency study of turbulent scaling intermittency using empirical mode decomposition and Hilbert spectral analysis. EPL (Europhysics Letters) 84(4), 40010 (2008)

25. Janas, P., Wlokas, I., Böhm, B., Kempf, A.: On the evolution of the flow field in a spark ignition engine. Flow, Turbulence and Combustion 98(1), 237-264 (2017)

26. Kaneko, M., Ikeda, Y., Nakajima, T.: Tumble generator valve (tgv) control of in-cylinder bulk flow and its turbulence near spark plug in si engine. SAE Transactions pp. 1635$1643(2001)$

27. Kapitza, L., Imberdis, O., Bensler, H., Willand, J., Thévenin, D.: An experimental analysis of the turbulent structures generated by the intake port of a DISI-engine. Experiments in fluids 48(2), 265-280 (2010)

28. Lumley, J.: The structure of inhomogeneous turbulence. Atmospheric Turbulence and Wave Propagation. AM Yaglom, VI Tatarski pp. 166-178 (1967)

29. Lumley, J.L.: Engines: an introduction. Cambridge University Press (1999)

30. Lumley, J.L.: Early work on fluid mechanics in the IC engine. Annual review of fluid mechanics 33(1), 319-338 (2001)

31. Marc, D., Borée, J., Bazile, R., Charnay, G.: Tumbling vortex flow in a model square piston compression machine: PIV and LDV measurements. Tech. rep., SAE Technical Paper (1997)

32. Müller, S., Böhm, B., Gleißner, M., Grzeszik, R., Arndt, S., Dreizler, A.: Flow field measurements in an optically accessible, direct-injection spray-guided internal combustion engine using high-speed PIV. Experiments in fluids 48(2), 281-290 (2010)

33. Ozdor, N., Dulger, M., Sher, E.: Cyclic variability in spark ignition engines a literature survey. SAE transactions pp. 1514-1552 (1994)

34. Peterson, B., Baum, E., Böhm, B., Sick, V., Dreizler, A.: High-speed piv and lif imaging of temperature stratification in an internal combustion engine. Proceedings of the combustion institute 34(2), 3653-3660 (2013)

35. Pope, S.B.: Turbulent flows (2001)

36. Qian, C., Wu, Z., Fu, C., Wang, D.: On changing El Niño: A view from time-varying annual cycle, interannual variability, and mean state. Journal of Climate 24(24), 6486$6500(2011)$

37. Rehman, N., Park, C., Huang, N.E., Mandic, D.P.: EMD via MEMD: multivariate noise-aided computation of standard EMD. Advances in Adaptive Data Analysis 5(02) (2013)

38. Reuss, D.L.: Cyclic variability of large-scale turbulent structures in directed and undirected ic engine flows. SAE transactions pp. 128-145 (2000)

39. Rothrock, A.M., Spencer, R.C.: The influence of Directed Air Flow on Combustion in Spark-Ignition Engine (1939)

40. Roudnitzky, S., Druault, P., Guibert, P.: Proper orthogonal decomposition of in-cylinder engine flow into mean component, coherent structures and random gaussian fluctuations. Journal of Turbulence (7), N70 (2006)

41. Sadeghi, M., Foucher, F., Abed-Meraim, K., Mounaïm-Rousselle, C.: Bivariate 2D Empirical mode decomposition for analyzing instantaneous turbulent velocity field in unsteady flows. Experiments in Fluids 60(8), 131 (2019)

42. Sakowitz, A., Mihaescu, M., Fuchs, L.: Flow decomposition methods applied to the flow in an ic engine manifold. Applied thermal engineering 65(1-2), 57-65 (2014)

43. Schmid, P.J.: Dynamic mode decomposition of numerical and experimental data. Journal of fluid mechanics 656, 5-28 (2010)

44. Sullivan, P., Ancimer, R., Wallace, J.: Turbulence averaging within spark ignition engines. Experiments in Fluids 27(1), 92-101 (1999)

45. Ur Rehman, N., Mandic, D.P.: Filter bank property of multivariate empirical mode decomposition. IEEE transactions on signal processing 59(5), 2421-2426 (2011) 
46. Voisine, M., Thomas, L., Borée, J., Rey, P.: Spatio-temporal structure and cycle to cycle variations of an in-cylinder tumbling flow. Experiments in fluids 50(5), 1393-1407 (2011)

47. Wang, G., Chen, X.Y., Qiao, F.L., Wu, Z., Huang, N.E.: On intrinsic mode function. Advances in Adaptive Data Analysis 2(03), 277-293 (2010)

48. Wiktorsson, M., Lindoff, B., Johansson, B., Soderberg, F.: Wavelet analysis of incylinder LDV velocity measurements. Tech. rep., SAE Technical Paper (1996)

49. Wu, C.H., Chang, H.C., Lee, P.L., Li, K.S., Sie, J.J., Sun, C.W., Yang, C., Li, P.H., Deng, H.T., Shyu, K.K.: Frequency recognition in an SSVEP-based brain computer interface using empirical mode decomposition and refined generalized zero-crossing. Journal of neuroscience methods 196(1), 170-181 (2011)

50. WU, Z., Huang, N.E., Chen, X.: The multi-dimensional ensemble empirical mode decomposition method. Advances in Adaptive Data Analysis (2009)

51. Yamakawa, M., Youso, T., Fujikawa, T., Nishimoto, T., Wada, Y., Sato, K., Yokohata, H.: Combustion technology development for a high compression ratio SI engine. SAE International Journal of Fuels and Lubricants 5(1), 98-105 (2012)

52. Zentgraf, F., Baum, E., Böhm, B., Dreizler, A., Peterson, B.: On the turbulent flow in piston engines: Coupling of statistical theory quantities and instantaneous turbulence. Physics of Fluids 28(4), 045108 (2016) 